\title{
MODIFICATION OF \\ THE GRAY EQUATION OF STATE \\ IN THE LIQUID-VAPOR REGION
}

David A. Young

April 15, 1974

Prepared for U'S. Atamic Energy Commission under contract Na W-7405-Eng-48

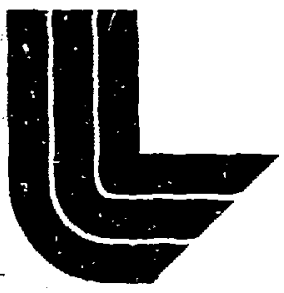

LAWRENCE LIVERMORE LABORATORY

University of Califomia / Livermore 

TID-4500, UC-34

Physics-General

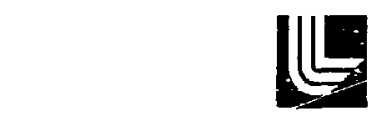

LAMRENCE LVERMOFE LABORATOAY

Univarsity of Cartoria/Livmare, Caftomia/,94550

\section{UCRL-51575 \\ MODIFICATION OF \\ THE GRAY EQUATION OF STATE \\ IN THE LIQUID-VAPOR REGION}

David A. Young

MS. date: April 15, 1974
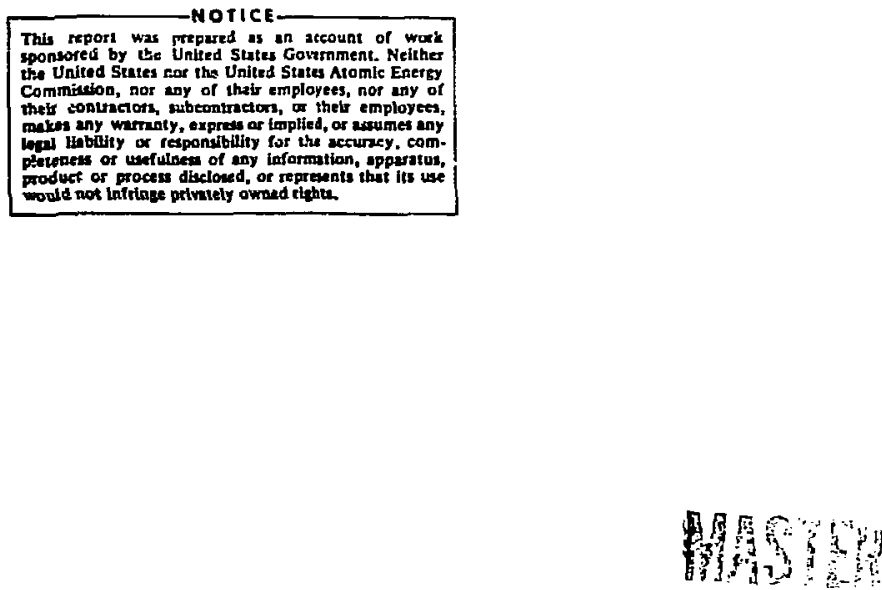

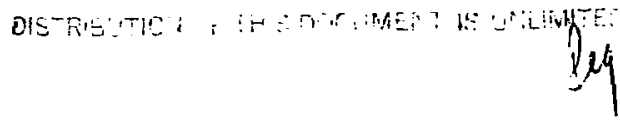




\section{IMDDIFICATION OF THE GRAY EQUATION OF STATE IN THE LIQUID.VAPOR REGION*}

\section{ABSTRACT}

A modification of the GRAY three-phase equation of state is described. This modification permits accurate calculation of the pressure in the mixed phase liquidvapor region. A listing of the computer code modification is given along with a listing of two-phase region paraneters for sixteen metals. Plots of the modified equation of state are also presented.

\section{INTRODUCTION}

When a porous solid metal is rapidly heated, it may enter a mixed phase liquid-vapor state at low pressure. In order for accurate predictions of such processes to be made, a three phase equation of state with an adequate representation of the equilibrium between liquid and vapor is nreded.

\section{THEORY}

At present the GRAY ${ }^{1}$ three-phase equation of state (EOS) uses a modified van der Waals theory for the liquid-vapor region, which gives rise to unphysical maxima and minima (van der Waals loops) in isotherns drawn in the pressure-volume plane (Fig 1). What are needed are the experimentally observed horizontal tie lines which connect the high density liquid with the low density vapor. The locus of tie line end points forms a dome-shaped curve in $\mathrm{p}-\mathrm{V}$ space, as shown in Fig. 1. The top of the dome is the critical point. The liquid and vapor states coalesce above the critical temperature $T_{c}$, and hence no changes in GRAY are needed above this temperature. The problem, then, is to modify GRAY in the two phase region so that a realistic equation of state results.

\section{CaLCULATIONS}

We begin with a computation of the critical point using GRAY pressure and energy isotherms, $P(V, T)$

\footnotetext{
Work perfomed under the auspices of the U.S. Atomic Energy Commission.

Partially supported by Headquarters, Defense Nuclear Agency under LACRO No. 74-824.
}

and $E(V, T)$. This is done by an iteration process which locates the lowest isothem which has no van der Waals loops (the critical isotherm), and also the volume at which $(\partial \mathrm{P} / \partial \mathrm{V})_{\mathrm{T}}=0$ (critical volume). Now we have the four critical constants $V_{c}, T_{c}, P_{c}=P\left(V_{c}, T_{c}\right)$, and $E_{c}=E\left(V_{c}, T_{c}\right)$.

The next task is to select a set of subcritical temperz tures for which energies and densities at the tie-line end points and pressures will be computed. We select an arbitrary number of equally spaced temperatures between $T_{c}$ and $T_{m o}$, the melting point parameter used in GRAY. These isotherms are used as base lines for interpolation so that the pressure can be computed for any volume and temperature within the two phase region.

For a more accurate inferpolation a larget set of temperatures is required, but this in turn would be more time consuming in the computation of the equation of state. We have chosen the minimum number of temperatures for each element cospatible with the desired snoothness and accuricy of the equation of state. The lowest acceptable tie line pressure is taken to be roughly 1 bar $\left(10^{-6} \mathrm{Mbar}\right)$, since pressures below this are gererally unimportant for the physical processes of interest, and they may be computed with sufficient accuracy by extrapolation from the 1 bar level.

Once the set of ye line temperatures is chosen, the corresponding pressures and equilibrium liquid and vapor densities ar: computed by the Maxwell construction. That is, a constant pressure tie line is drawn through the van der Waals losp so that the $\mathrm{p}-\mathrm{V}$ area abowe the line is equal to that below. Altematively, we seek a pressure $\mathrm{P}_{\mathrm{e}}$ such that

$$
\int_{v_{\min }}^{v_{\max }}\left(p-p_{e}\right) d v=0
$$

when the integral is taken from the liquid volume $V_{\text {min }}$ to the vapor volume $V_{\max }$. This calculation is done by an iteration procedure in which a given estimate of $\mathrm{Pe}_{e}$ is used in the evaluation of the above integral, and the sign of that integral is used to determine what the next estimate of $p_{e}$ should be. Once $V_{\min }$ and $V_{\max }$ at

\footnotetext{
1. E.B. Royce, "GRAY, A Three-Phase Equation of State for Metals", Lawrence Livermore Laboratory Rpt. UCRL-51121 (1971).
} 
temperature $T$ are obtained, then the energies $E\left(V_{\text {min }}, T\right)$ and $\mathrm{E}\left(\mathrm{V}_{\max }, \mathrm{T}\right)$ are computed, and the calculation proceeds to the next temperature.

This preliminary calculation is done once for each material, and the computed critical point and tie line data are then stored. Listings of the critical point and tie line calculations are shown in Appendix A. The critical point and tie line data are shown in Table I for the sixteer. metals in the GRAY report. ${ }^{1}$ The data needed for the equation of state calculation can either be computed directly and stored, or read in and stored. Of course, any desired variation in the calculation described above or any data for a new material will require a fresh calculation.

Hydrodynamic calculations require pressure as a function of volume ard energy, $P(V, E)$. The pressure will be calculated directly from GRAY everywhere except within the boundary of the liquid-vapor region. The boundar: is constructed from line segments in the E-V plane which join the stored tie line end points together. The boundaries joining the $\mathrm{E}=0$ axis to the lowest liquid and vapoi tie line and points are linear in density rather than volume. The solid-vapor sublimation curve is disregarded and the iiquid-vaper boundary is extrapolated to a volume $1.04 \mathrm{~V}_{0}$ at $\ddot{\boldsymbol{c}}=0$. This is done to prevent the appearance of liquid under tension in the model.

The first task of the modification in GRAY then consists of determining whetier the $(E, V)$ point of interest is within the two-phase boundary, and if so, detemining between which two tie lines the point lies Once this is done, the temperature is estimated by interpolation, assuming that the energy varies linearly with temperature. Finally the pressure is determined by interpolation using the proportionality between enp and $1 / T$ predicted by the Clausius-Clapeyron equation. This coding, which may be inserted into the GRAY subroutine, is listed in Appendix B.

Plots of $\mathrm{p}-\mathrm{V}$ isotherms for the six teen GRAY nietals are shown in Appendix C. Here the tie lines predicted by interpolation procedures are clearly shown, and some roughness due to the approximations used in these procedures also agpears.

\section{ACKNOWLEDGEMENT}

We acknowledge the support of Mr. Don Kohler of DNA, the technical noniter for the DNA-sponsored portion of this project. 


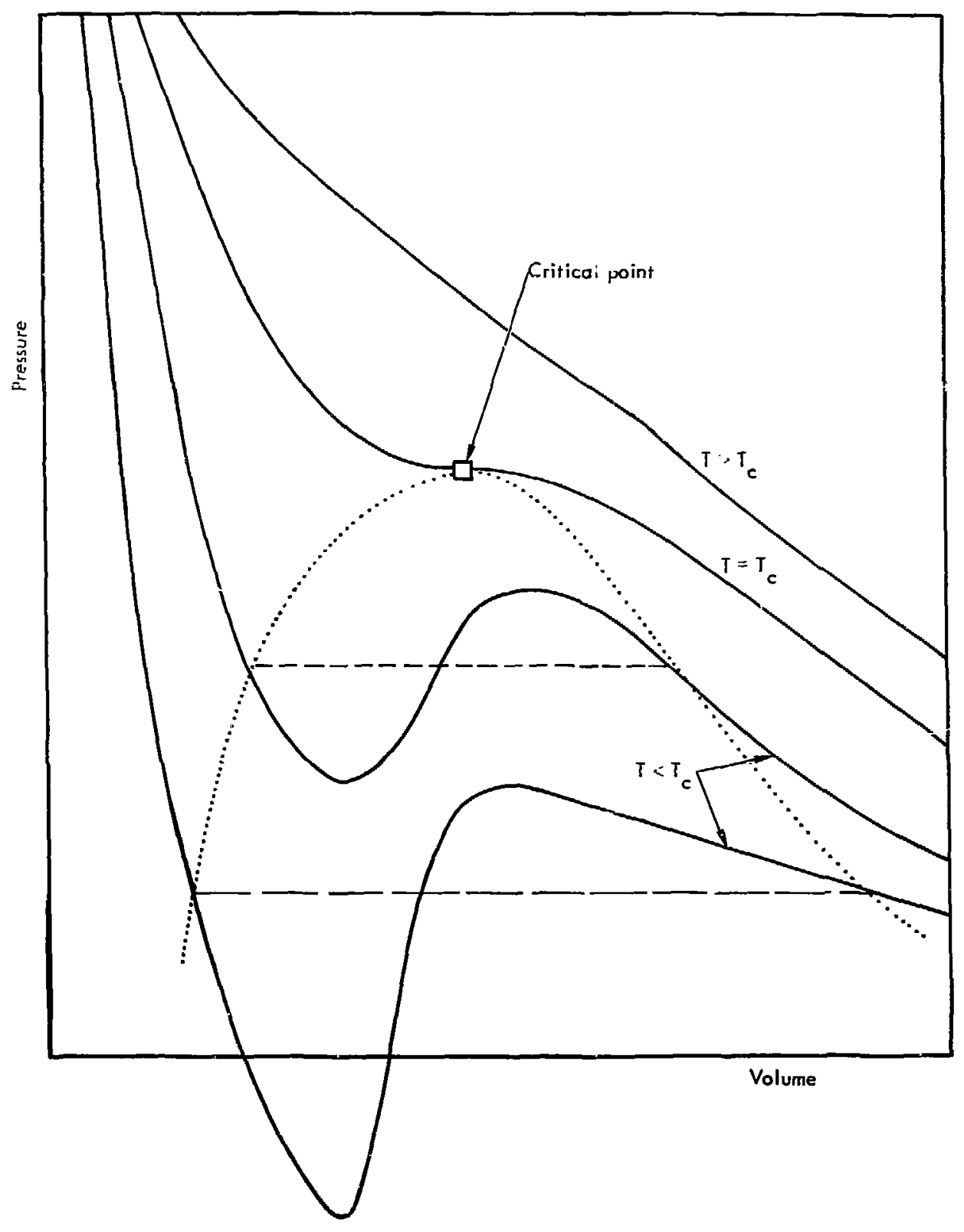

Fig. 1. A echematic diagram of GRAY isotherms in the $\mathrm{p}-\mathrm{V}$ plane. The subcritical $\left(T<\mathrm{T}_{c}\right)$ isotherms exhibit nonphysical van der Weals loops, which are to be roplaced by constant prespure tie lines (dashed lines). The :ocus of the the line end posnts forms the domashaped coexistence curve (dotted curve), with the crittcal point at the top. 
Table I

Here the critical point and tie line parameters are listed for each of the sixteen metals of the GRAY report. The first line of numbers after the name of the metal lists the four critical point parameters, which are defined as follows: TC is the criticai temperature (degrees $\mathrm{K}$ ), VC is the critical volume $\left(\mathrm{cm}^{3} / \mathrm{g}\right.$ ), $\mathrm{PC}$ is the critical pressure (Mbar), and EC is the critical energy $\left(\mathrm{Mbar}-\mathrm{cm}^{3} / \mathrm{g}\right)$. The tie line parameters follow the critical point data and are defined as follows: TEMP is the tie line temperature (degrees $\mathrm{K}$ ), VMX is the volume of the vapor end of the tie line $\left(\mathrm{cm}^{3} / \mathrm{g}\right)$,
VMN is the volume of the liquid end of the tie line $\left(\mathrm{cm}^{3} / \mathrm{g}\right)$, EVMX is the energy at VMX and TEMP $\left(\mathrm{Mbar}^{\mathrm{cm}} \mathrm{cm}^{3} / \mathrm{g}\right), \mathrm{EVMN}$ is the energy at VMN and TEMP $\left(\mathrm{Mbar} \cdot \mathrm{cm}^{3} / \mathrm{g}\right)$, and PRES is the tie line pies. sure (Mbar). The index JAA is the number of equally sfaced subcritical temperatures (including $\mathrm{T}_{\mathrm{mo}}$ ) for which tie line data are to be cosputed. Here JAA $=\mathrm{JAB}-1$, where JAB is the index used in the tie line generation code in Appendix A. The indey JMAX is the total number of tie lines used, including the critical point.

BERYLLILY

$$
J A A=10 \quad J M A X=10
$$

TC VC FC EC

$$
\text { 1.132BE+\{4 } \quad 1.88 E Z E+\& Q \quad 2.1523 E-02 \quad 4.1623 E-01
$$

\section{TEMP}

1. $8917 E+G 4$

$9.9065 E+[.3$

$8.8957 \equiv+03$

$7.8849 \succeq+03$

$6.8740 E+03$

5. $3632 E_{-}+03$

4. $8524=+[C 3$

3. $8416 E+23$

$2.83[$ A $E+03$
VMX

4. 1EETE+ 00

$E, 6 E 67 E+0 \bar{C}$

$1.1111 E+\eta 1$

1. $6 \overline{C E} 7 E+J 1$

$3.3333 t+01$

5. $0000 \div+31$

1. $6 E E 7 E+08$

5. $0200 E+12$

ร. JULCE+CZ
V'AN

1. 1E2 $3 E+0 C$

1. $82 D L E+D C$

$9.434[E-01$

8. $9285 E-01$

8. $547 E E-J 1$

8. $3333 E-01$

3. CCOJE-D1

7. $3529 \equiv-11$

E.71145-01
EVix

$4.7595 E-01$

$4.7516 E-01$

$4.7190 E-01$

4. $6077 E-01$

$4.5037 E-01$

4. $3480 E-01$

$4.2138=-01$

4. $05095-01$

3. $89115-01$
EVMN

$3.2280 E-01$

2. $821 \in E-01$

2.5155E-01

$2.259 \mathrm{SE}-01$

2.0337E- 01

$1.8378 \bar{E}-01$

$1.6128 E-01$

$1.2966 E-01$

$9.5140 E-02$
PRES

1. 4235E-112

$9.2249 E-03$

5. $6057 E-03$

$3.1083 \mathrm{E}-03$

$1.5338 E-03$

$6.0738 \mathrm{E}-04$

$2.3285 E-04$

4. $9149 E-05$

$3.9372 E-0 E$

MAG'ISIUN

$\sin A=1 \mathrm{i} \quad J M \Delta x=9$

TC

$v c$

FC

EC

$7.4169=+03$

1. $95 E G E+0 ?$

4. $28605-03$

$9.9125 E-02$

TE:P

VMX

V:1N

EVMX

EVMN

PRES

\section{$6.7902 E+0.3$ \\ $6.1 E 35 E+[.3$ \\ $5.5368 E+C^{3}$ \\ 4. $9101 E+0.5$ \\ 4. $2334 \hat{E}+[3$ \\ $3.6567 E+[3$ \\ 3. $0301 \equiv+03$ \\ $2.4034 E+[3$}

\begin{abstract}
$4.1 E \in 7 E+] E$
$7.1429 E+\bar{O} \mathrm{O}$

$1.1111 E+31$

2.00CUE+01

3. $3333 \equiv+01$

1. OCOOE+IE

2.000OE+ D2

1. $02005+03$
\end{abstract}

1.1111 $\overline{0}+00$

$1.12545-51$
9.7गR7E-ü 1

Q. $0[0.0-71$

$8.5470 \equiv-71$

ม. $1567 \equiv-91$

7. $9365 E-j 1$

7. $3125 \equiv-01$

$7.4 E 27 \equiv-01$
1. $1377 \equiv-01$

$1.1230=-01$

1.1917 $5-51$

1. $7580=-01$

1. $2375 E-21$

$9.9799 \equiv-02$

$5.5365 \equiv-02$
$7.45: 9 E-02$

$6.4128 E-02$

$5.6595 E-02$

3. $0315 \bar{L}-02$

$4.4784 E-02$

$3.9861 E-02$

$3.5441 E-02$

$2.9469 E-02$
$3.2589 E-03$

2. $0652 E-03$

1.2210E-03

6. 5951E-04

$3.1074 E-04$

$1.1466 E-04$

4. $4717 E-05$

$7.9731 E-0 E$ 
ALUHI AUN

$J A A=10 \quad J M A X=9$

TC

VC

FC

EC

$1.1533 E+04$

TEMP
1. $2118 \bar{E}+00$

VHX

3. $0303 E+00$

$5.2632 E+00$

$9.0909 E+00$

$1.6 \leqq 67 E+U 1$

$3.3333 E+01$

$1 \cdot 1111 E+02$

$3.3333 E+02$

$2.0000 E+03$
1. $0468 E-02$

EVMX

1. $6646 E-01$

1. $6642 E-51$

1. $6307 E-01$

1. $5824 E-01$

1. 3405E-01

5.0251E-01 1.5255E-01 5.4898E-02

$4.8780 E-01 \quad 1.4569 E-01 \quad 4.7095 E-02$

$4.7847 E-01 \quad 1.4028 E-01 \quad 4.0142 E-02$

$1.4590 \mathrm{E}-01$

EVMN

PRES

$1.0529 E-01$

$6.6685 E-03$

$8.7514 E-02 \quad 3.9305 E-03$

$7.4444 E-02 \quad 2.1766 E-03$

$6.3819 E-02 \quad 1.0529 E-03$

$4.2583 E-04$

$1.3457 E-04$

$3.6220 E-05$

4. $E \subseteq 4 B E-01$

$3.367 \mathrm{BE}-02$

$4.1429 E-06$

TITAN IUM

$J A A=10$ JM $A X=9$

TC

vC

$P C$

ES

$1.4466 E+04$

$$
7.5718 \Xi-01
$$

1.17EOE-D2

$1.2300 \bar{E}-01$

TEMP

VHX

VMN

EVMX

EVMN

PRES

$1.3246 E+04$

$1.2025 E+04$

1.0805E+04

$9.5839 E+03$

B. $3632 E+03$

$7.1426 E+03$

$5.9219 E+03$

4. $7013 E+03$
$1.9231 E+0 C$

$3.3333 E+00$

5. $8224 E+0 C$

1. $1111 E+01$

2. 5 COOE+ 01

5. $0000 E+01$

1. $6 E 57 E+02$

$1.0000 r+03$
4. $3103 E-31$

3.7037E-01

$3.4014 E-01$

$3.2259 E-01$

3. $1250 E-01$

3. $0303 \leq-01$

2. $8902 E-91$

$2.7322 E-31$
$1.3790 E-01$

1. 3715E-01

$1.3419 E-01$

$1.3015 \equiv-01$

1.2561E-01

$1.2057=-01$

1. $1572 E-C 1$

$1.1098 \equiv-01$
$9.1128 \bar{E}-02$

$7.6 .923 E-02$

6. $6851 E-02$

$5.8992 E-42$

$5.2637 E-02$

$4.620 \mathrm{JE}-02$

3.871EE-02

$3.0642 E-02$
$7.5217 E-03$

$4.5300 E-03$

$2.5253 \mathrm{E}-03$

$1.2674 E-03$

$5.4393 E-04$

$1.8974 E-04$

$5.5024 E-05$

$7.8376 E-06$ 
STA INLESS

$J A D=10 \quad$ JMAX $=10$

TC

VC

FC

EC

1. $4721 E+04$

4. 52.39E-01

$1.7495 E-02$

1. $0011 E-01$

TEMP

VMX

VMN

EVIYX

EVMN

PRES

$1.3487 E+04$

1. $2253 E+04$

1. $1019 \mathrm{E}+04$

$9.7846 E+03$

0.5505E+03

7. $3164 E+03$

$6.0823 E+03$

$4.8482 E+03$

3. $E 141 E+03$
1. $0526 E+00$

1. $7857 E+00$

3. $0303 E+00$

$5.2632 E+00$

1. OCOOE+D1

2. $5600 E+01$

5. OQOOE+01

2. $5000 E+0 E$

2. 5 COOE +0 3

\begin{abstract}
2. $[954 \bar{E}-31$
1. $1037 \equiv-01$

1. $49635-01$

$[.325 \in E-71$

1. $0702 E-01$

1. $9020 \mathrm{E}-01$

1. $0347 \equiv-01$

$1.0011=-01$

1. $8382 E-01$

$1.7889 \vec{E}-01$

$1.7513 E-01$

1. E5B4E-J1

$9.95345-02$

c. 5495E-02

$0.1204 \Xi-02$

8. $7175 E-02$

8. $3289 E-02$
\end{abstract}

$7.6220 E-32$

$6.462 \mathrm{GE}-02$

$5.5742 E-02$

$4.8453 E-02$

4.227 गE- 12

$3.6925 E-32$

$3.2155 \mathrm{E}-02$

2.783 GE - U2

$2.20 y 9=02$
1. $1674 E-02$

$7.373 I E-03$

$4.33+2 E-03$

2. 31 C $8 E-03$

1. $0725 E-03$

4. $0490 \mathrm{E}-04$

1.081JE-04

2. $4321 E-05$

$1.9346 E-0 E$

NICKEL

$J A A=10 \quad J M A X=10$

TC

VC

$$
3.9[1 \varepsilon E-u 1
$$

VAN

1. $ᄃ 2 f 2 E-b 2$

TEMP

VMX

EVMX

2. $2[41 \Xi-1] 1$ 1. $9.841 \Xi-\mathrm{J} 1$

1. $116 \equiv-\overline{1} 1$

1.7 $375-U 1$

1. E2 SปE-J 1

$1.5743 E-01$

$1.540 d E-01$

1. $5033 E-31$

1. $41445-01$
1. $0730=-01$

1. $0643 E-01$

1. $C 373 E-[1$

1. $0031 E-01$

$0.6389 \equiv-0 \bar{c}$

$0.2205:-02$

$8.8170=-02$

8. $4170 \bar{z}-0 \bar{C}$

8. $0+10 E-0 E$
EC

$9.7229 E-02$

EVMN

$P$ P:ES

$7.3539 \overline{9}-02$

$0.22 J 7 E-02$

$5.35495-02$

$+.6497 \equiv-02$

4. $0550 E-02$

$3.5427 E-02$

3. $098.3 z-02$

$2.6455 E-02$

2. $0253 E-02$
$1.2734 E-02$

7. $9557 E-03$

4. $0163 E-0 ?$

$2.42 ? 5 E-[3$

1. 1 L $13 E-03$

4. C23DE-D 4

1. $035+E-04$

$2.1691 E-05$

1. $4551 E-D E$ 
MONEL

$J A A=10 \quad J M A X=10$

TC

ve

PC

E C

$\begin{array}{lrrrr}1.3177 E+04 & 4.0885 E-01 & 1.60 E 3 E-02 & 8.1996 E-02 \\ \text { TEMP } & \text { VMX } & \text { VMN } & \text { EVYX } & \text { EVMN }\end{array}$

PRES

1. $2087 E+04$

$9.434 D E-D 1$

$2.4510 E-21$

Q. $0365 \equiv-02$

$6.2863 E-02$

1. $0996 E+04$

2. $1196 E-\square 1$

8. $970 \square E-02$

1. $5 E 25 E+30$

1. $9342 E-i 1$

8. $8144 E+03$

E31EE+OO

8.7677E-02

1. $2149 E-01$

Q. $4876 E-02$

$7.7237 E+03$

$1.73015-01$

Q. 1807E-02

$6.6329 E+03$

1. $E 722 E-01$

7. $8464 E-02$

$5.5422 E+03$

2. OCUCE+D1

5. OCOOE+J1

1. E2GOE-01

7. 5055E-02

4. $4515 E+03$

$2.000 J E+02$

1. 59495-01

$7.1738 E-02$

3. $3607 E+03$

1. $6 E E 7 E+03$

1. $5175 E-01$

6. $8561 E-02$

$5.3560 E-02$

$4.6385 E-02$

$4.0483 E-02$

$3.5438 E-02$

3.108 4E-02

2.7183E-02

$2.3731 E-02$

1.9257E-02

$1.0835 E-02$

$6.9355 E-03$

4.1463E-03

$2.2606 E-03$

1. $0817 \mathrm{E}-\mathrm{J3}$

4.2715E-04

$1.2593 E-04$

$2.8717 E-05$

2.7584E-06

COPPEF

JA $A=10$ JMAX $=10$

TC

vC

FC

EC

1. $225 E 5+34$

$3.6489 \Xi-01$

1. $5711 \overline{5}-02$

$6.0804 E-02$

TE:AP

VMX

EVAX

EVMN

PRES

$1.12105+C+9.5+70 E-01$

2. 1978E-J1 E.9981E-02

1. $01 \in 3 \equiv+0+$

$1.4493 E+9 \overline{\mathrm{O}}$

1. $\subseteq 157:-D 1$

7. $.8855 E-02$

4.5527E-02

1.0405E-02

$3.1154 i+03$

$2.5200 E+00$

1.7E59E-01

7. $0279 E-0 ?$

$3.8991 E-02$

1. ET2ZE-J1 E. B7Э7E-02

$7.0232 E+[3$

$4.347 ? E+0$

1. E $T 77 E-J 1$

$6.6907 \equiv-82$

$5.9765 E+03$

2. $02005+21$

$6.47705-92$

$3.4199 E-02$

$3.028 E E-02$

1. $5625 \div-01$

2. $69175-02$

$2.3914 E-02$

5. $0[0] 0+1] 1$

E. $2433=-02$

$3.9333 E+[3$

1. $5291 \equiv-31$

E. $0011 \equiv-02$

2.1165E-02

$2.5500 E+32$

1. $45+3 E-31$

$1.8309 \varepsilon-02$

1. $40455-01$

$5.7514 \bar{\Xi}-02$

1. $4044 E-02$

E. 5348E-03

$3.8239 E-03$

$2.0287 E-03$

S. 3392E-0 4

$3.4743 E-14$

$9.1616 \mathrm{E}-05$

$1.8076 E-05$

$1.1381 E-D E$ 
NIOJIUM

$J A A=10 \quad J M A X=10$

TC

VC

PC

EC

$2.4572 E+\overline{4} 4$

4. $3271 \div-01$

$1.8231 E-02$

$1.2547 E-01$

TEMP

$\sin x$

VMN

EVYYX

EVMN

PRES

2. $24 E 8 E+04$ 2. $0364 E+84$ 1. $8260 E+04$ 1. $6155 E+04$ $1.4051 E+04$ 1. $1947 E+[4$ $9.8427 E+[3$ $7.7384 E \div G 3$ $5.6342 \mathrm{E}+[3$
1. C5ZEEF50 $1.8182 E+04$ $3.2256 E+00$ $5.8824 E+00$ 1. $25005+01$ $3.3333 E+01$ 1. $1111 E+02$ 5. $0300 \mathrm{O} E+02$ $1.0000 E+04$
2.50DJE-01 Z. $1231 E-J 1$ 1. $\subseteq 157 E-\{1$ 1. $7857 E-11$ 1.E $E 49 E-01$ 1.E $313 E-01$ 1. $5549 \vec{E}-81$ $1.53375-01$ 1. $43 E 8 \vec{E}-J 1$
$1.3280 E-01$ 1.2951E-01 1. $2298 E-01$ 1. $1668 E-01$ $1.1036 E-01$ 1. $0415 \bar{z}-01$ Q.8197E-02 $0.2733 E-02$ $8.7797 \bar{c}-02$
$9.5856 E-02$

7.9690E-02 $6.7025 E-02$ $5.6743 E-02$ 4.8132E-02 4.0867E-02 $3.4934 E-\cap ?$ $2.8429 E-02$ 2.0787E-02
$1.1918 E-02$

$7.3063 E-03$

4.1205E-03

$2.0749 E-03$

8. $9018 E-04$ $2.9821 E-01$ $7.1443 E-05$ $1.2106 \varepsilon-05$ 4.8219E-07
TANTALUM

$J A A=11 \quad J M A X=11$

TC

$\because c$

$2.242 E E-J 1$

$P C$

1. $2859 E-02$

$2.5547 \equiv+04$

$\operatorname{vin} x$

VYN

EVMX

$7.3034 \bar{c}-02$

$7.1117 \equiv-02$

$6.84112=-02$

$5.5427 E-02$

E. 2235E- 02

E. $9191 E-02$

$5.518+E-02$

E.3311E-02

$5.0669 \equiv-02$

$4.8253 E-0 \bar{C}$
EC

$6.9179 E-02$

EVMN

$5.4391 \bar{c}-02$

4.6206E-02

$3.9681 E-02$

$3.4242 E-02$

$2.9644 E-02$

2.571 BE- 02

$2.2440 E-02$

$1.9519 E-92$

$1.6144 E-02$

$1.2348 E-02$
PRES

$1.3026 E-02$ 8.5704E-03 $5.305 B E-03$ $3.0368 E-03$ 1. $5652 E-03$ E. $9689 E-04$ 2.5058E-04 E. 4971E-05 $1.3360 E-05$ 1. $0494 E-0 E$ 
TUNGS TEN

$J A A=11$ JMAX $=11$

TC

vC

FC

EC

$2.6037 E+04$

$1.764 E E-01$

$2.3899 E-02$

$7.0744 E-02$

TEMP

VMX

V.N

EVMX

EVHN

PRES

$2.4081 E+C 4$

2. $2125 E+04$

$2.0169 E+04$

$1.8213 E+04$ 1. $6257 \mathrm{E}+04$ $1.4301 \mathrm{E}+04$ $1.2344 E+04$ $1.0388 E+04$ $8.4322 E+03$ $6.4761 E+03$
4. $0 \subseteq B 4 E-D 1$

6. $8493 E-01$

1. $1364 E+00$

1. $9808 \mathrm{E}+00$

$3.5714 E+90$

$7.6923 E+00$

1. $6 E 67 E+01$

5. $0000 E+01$

$2.5000 E+02$

$2.5000 \Xi+0 ?$
1. $0331 E-11$

8. $7873 E-32$

7. $9365 E-12$

7. $3855 E-32$

7.0126E-02

$6.8 \in 34 E-92$

$6.7114 E-02$

E. 5020E-02

E. 2539E-02

c. C095 E-ก ?
$7.524 \mathrm{~J} \equiv-02$

$7.3553 E-02$

$7.0964 E-12$

$\epsilon .80275-02$

E. 4945E-02

E.1999E-02

5.8836E-02

5.60565-02

$5.34295-02$

$5.1038 \varepsilon-0 \overline{2}$ 5.5210z-02 $4.6823 E-02$ $4.0270 E-02$ $3.490 B E-02$ 3.0457E-02 $2.7 \cup 22 E-02$ $2.3577 E-02$ 1.9861E-02 $1.599 \mathrm{DE}-02$ 1. $2214 E-02$
1.6294E-02 $1.0563 E-02$ $6.4337 E-03$ 3.6170E-03 1. $8276 \mathrm{E}-03$ ?. $9780 E-04$ 2.8077E-04 7.0519E-05 1.3554E-05 1. D055E-06

GOLD

$J \triangle A=11$ JMAX $=10$

TC

vC

FC

E.C

$1.8797 \bar{E}+34$

$1.72 B 25-01$

1. $E 4295-02$

$3.3677 E-02$

TEMP

VMX

VMN

EVMX

EVIN

PRES

1. $7267 E+64$

1. $5737 E+04$

$1.4208 E+04$

1. $2678 E+04$

$1.1148 E+04$

$9.6185 E+03$

8. $0888 E+03$

6. $5591 E+03$

5. $0294 E+0.3$
4.0486E-01 1

6. $8493 E-01$

$1.1494 E+0 C$

2. $0408 E+00$

4. OCOOE+ DO

$9.0909 E+00$

$2.5 C 00 E+01$

$1 \cdot 1111 E+02$

5. $0000 E+0 E$
$1.0277 E-31$

ง. $8731 E-02$

$3.1 \cup 37 \ddot{c}-0 z$

7. E161E-0E

$7.2933 E-92$

$7.0423 E-132$

E. $8537 E-i j z$

E. $7247 E-02$

6. $E 05 . j E-02$
$3.7348 \mathrm{c}-02$

$3.7375 E-0 Z$

$3.7184 E-02$

3.6171E-02

$3.4970 E-32$

3. $3659 E-0 \tilde{C}$

3. 2295E-02

$3.0943 E-02$

2. $963 \cup E-02$
$2.5207 E-02$

2.124.9E-02

$1.8254 E-02$

$1.5828 E-02$

1.3777E-02

$1.1986 E-02$

$1.0385 E-02$

$8.9317 \bar{c}-33$

$7.4315 E-03$
1.1008E-02

E. $9990 E-03$

4. $1583 E-03$

$2.2551 E-03$

1.0759E-03

$4.2498 E-04$

1. $2517 E-04$

$2.3185 E-05$

$2.5477 E-06$ 
JA $A=11$ JMAX $=9$

TC

VC

$$
2.713 \varepsilon \varepsilon-01
$$

?.7027E-0 03

EVMX

1. 3131E- $[2$

1. $3193 \mathrm{E}-0 \bar{C}$

$1.2987 \equiv-02$

1. 2665E-B2

1. $2269 \equiv-02$

$1.13 .33 E-0 E$

$1.1360 E-02$

1. $0911 E-02$
EC

1.1552E-02

EVMN

PRES

$8.5541 E-03$

$7.2017 E-03$

$6.203 .3 E-03$

5.395 JE -03

$4.7161 \bar{E}-03$

$4.1171 t-03$

$3.5811 \bar{E}-\mathrm{i} 3$

$3.092 \mathrm{JE}-03$
$2.4717 E-03$

$1.5674 E-03$

9.2995E-04

$5.0447 E-04$

$2.4131 E-04$

$9.5812 E-05$

$2.8653 \varepsilon-0 \mathrm{~g}$

E. $2510 E-0 E$

THO IUM

$J A A=11$ JMAX $=10$

TC

vC

PC

EC

$2.0701 E+04$

$3.5715 E-01$

$7.44185-03$

$4.37075-02$

TEMP

VMX

VMN

EVYX

EVMN

PRES

1. $9036 E+04$

B. $0 E 45 E-01$

2. 1142E-01

4. $5197 \bar{E}=02$

$3.436 E E-02$

$5.12 E 7 E-03$

$1.7372 E+04$

$1.5707 E+04$

$1.4042 \varepsilon+04$

$1.2378 E+04$

1. $0713 E+04$

9. $0485 E+03$

$1.3158 E+00$

1.7953E-J 1

4. $3403=-02$

$2.1739 E+\tilde{U} \mathrm{E}$

1. $E 129 E-01$

4.1249E- 02

$1.4903 E-51$

3. $8951 E-02$

$7.1429 E+00$

1. $4025 E-01$

$3.6578 E-0 \bar{c}$

1. $3359 E-31$

3. $44265-02$

1. 2 CB $7 E-31$

$3.2299 E-02$

$3.3333 E+21$

$7.3839 E+03$

1. $4286 \mathrm{E}+0 \overline{2}$

1. $2 E 0 J E-01$

$3.0341 \Xi-02$

$2.8897 E-02$

$2.4444 E-02$

3. $3484 E-03$

2.0687E-02

$1.7485 E-02$

$1.4745 E-02$

$1.2402 E-02$

$1.039 \exists E-02$

2. $0434 E-03$

1. $1401 E-03$

5. $6252 E-04$

$2.3265 E-04$

$7.332+E-05$

1. $1843 E-01$

$2.9551 E-02$

$1.7313 E-05$

2. $0224 E-0 E$ 
IUL 3E FRY

$J 4 A=11$ JHAX $=10$

TC

VC

PC

5C

$2.0338 E+04$

$2.1427 \equiv-01$

1. $2 E \in 7 E-02$

$4.0268 E-02$

TEMP

VMX

VMN

EVHX

EVHN

PRES

1. 851 तE +04 1. $68985+04$ 1. $5179 \mathrm{gE}+04$ 1. $3459 E+04$ 1. $1739 E+04$ 1. $0019 E+04$ 8. $2992 E+03$ $6.5794 \vec{E}+C ?$ 4. $8596 E+03$
$4.9751 E-51$

B. $4 C 34 z-01$

1. $42 \Delta E E+00$

$2.5 E 41 E+00$

5. $000 \mathrm{OD}+0 \mathrm{C}$

1. $2500 E+01$

$3.3333 E+81$

1. $6 E 67 E+0 \bar{C}$

1. $6 E G 7 E+03$
1. 2E10E-01

1. $C 741 E-01$

9. E712:-D2

8. $9767 E-0 Z$

3. $4746 E-02$

8. $11035-02$

7. B431E-0?

7. E3 $3 E \check{c}-02$

7. $44 J 5 E-02$
4. $2211 E-02$

4. $0686 E-02$

3. 8695E-02

$3.6546 E-02$

3. $4361 E-02$

$3.2246 E-02$

3. 0201E-02

$2.8320 \leqq-02$

$2.6519 E-02$
3.0854E-02

$2.5544 E-02$

$2.1261 E-02$

$1.7704 E-02$

1. $4676 E-0 ?$

1.2097E-02

$9.8874 E-03$

$7.9763 E-03$

$6.2721 E-03$
6. 5247E-G3

5.4092E-03

3.1799E-03

1. 5865E-0z

$7.7338 E-04$

$2.8511 E-04$

$7.4596 E-05$

1. 3267E-05

$9 \cdot 4074 E-07$
URANIUM

$J A A=11 \quad J M A X=10$

IC

ve

FC

$1.2831 E-02$

$1.8759 \div+04$

1. $.3 \geq 1 \in \Xi-01$

$\checkmark \div$ N

EVMX

TEYP

Vit $x$

$1.0741 E-11$

$9.1743 E-9 \overline{6}$

$8.2989 E-02$

$7.7399 \equiv-12$

$7.347 j E-0 \hat{E}$

7. $0 E 215-52$

$6.854 \mathrm{JE}-\mathrm{J} 2$

E.7069E-02

6. $5147 \mathrm{E}-32$
$3.6530 E-02$

3. $5591 \Xi \cdot 02$

3. $4291 . E-02$

$3.2685 \mathrm{E}-\mathrm{C2}$

3. 1.J2 OE 02

$2.9345 E-0 E$

$2.7745 \equiv-02$

2. $6224 E-02$

$2.4 B 33 E-62$
EC

$3.4251 E-02$

EVMN

PRES

2.6058E-02

$2.1675 E-02$

1.822 OE- 02

$1.5384 E-02$

1. $2989 \mathrm{E}-02$

1. $09405-02$

$9.1714 E-03$

$7.6437 E-03$

$6.1259 E-03$
8.5609E-03

$5.3718 E-03$

3.1525E-03

$1.6685 E-03$

7.6812E-04

$2.8741 E-154$

7.7622E-05

$1.4276 E-05$

1.2119E-06 


\section{APPENDIX A}

The following listing contains the critical point and tie line calculations required for the computation of the equation of state in the liquid-vapor region. This was introduced as a subroutine in the WONDY hydrodynamic code at livermore. COMMON statements are needed to inin common variables in the GRAY EOS subroutine and in this subroutine. Aiso, in cases where several different materials may enter the two phase region, the critical point and tie line parameter (Table l), including JAA and JMAX, mus! be subscripted according to the material layer being described. The index JAA must be given a value, as required by the first statement in the listing.

Attached to this listing are two subprograms for pressure (PT) and energy (ET) according to the GRAY EOS. These subprograms contain only the liguid and vepor portions of GRAY, since the solid EOS is not needed in the two-phase construction. These subprograms also need COMMON statements joining them with the GRAY subroutine.

\section{MAIN SUBROUTINE}

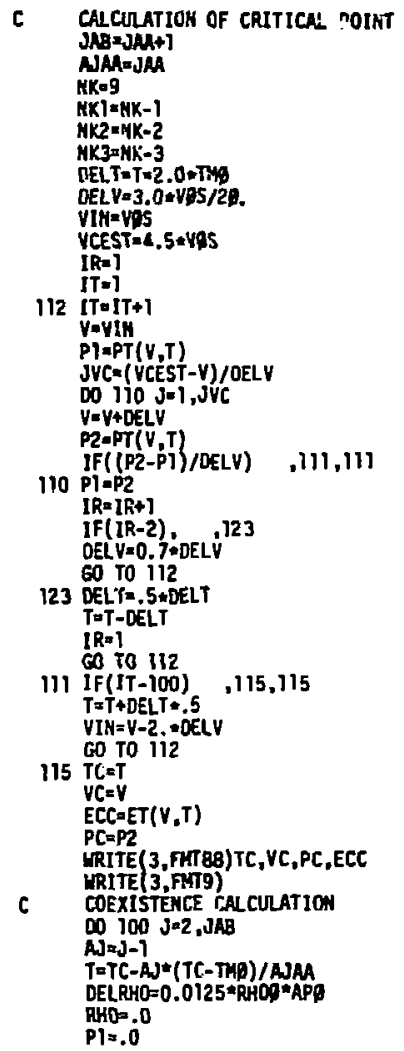

KII 1

$0020 \mathrm{k}=1,100$

RHO $=$ RHO $\rightarrow$ DEL RHO

$V=1.0 / 240$

P2-Pr (Y,T)

IF ((PZ.LE.PI),ANE. (K).EQ.2))GO TO 20

IF(P2.GT.PI)GO TO 12

$P H A X=P]$

$K \mid=2$

EO TO 20

12 IF(KI.EQ.1) 00 To 20

PHIM=P2

$60 \mathrm{TO} 16$

$20 \mathrm{Pl}=\mathrm{P2}$

16 PEQ, $5 *$ (PMAX + PHIM $)$

IF(PEQ.GT.0)GO TO 22 $P E Q=, 5$ PHAX

22 Do 25 KIT= 1,50

DO $300 \mathrm{~K}=1, \mathrm{MK} 2$

00400 L-1,9

ALEL

$V=(10, \cdots($ HK-K) $) / A L$

PHO=l. $N$

$P \mid=P T(V, T)$

IF(P1.GE.PEQ)GO TO 41

$4 C O$ CONTIKUE

300 CONTINUE

$K=N k 1$

DO $45 \mathrm{~L}=10,2500$

$A L=L$

$V=100.0 / \mathrm{AL}$

$\mathrm{RHO}=1 . N$

$P I=P T(Y, T)$

IF(PI.GE.PED)GO TD 4 ]

45 CONTIMUE

4) RHOHTH=PHO

SUAR $=.0$

$I N=]$

IF(K.GT.NK3) CO 10 53

DO 55 XK=K, HK3

DELRHO=1.0 $(10.0 *$ (HK-KK))

DO $60 \mathrm{LL}=\mathrm{L}, 10$

$A L=L I$

PHO=AL"DELRHO

$V=i .0 /$ RHO

$P 1=P T(V, T)$

$F=P]-P E Q$

SURT $=S U F R+F=D E L R H O / R H O=2$

IF(F.LE. O.) IN=? 
MAIN SUBROUTINE (continued)

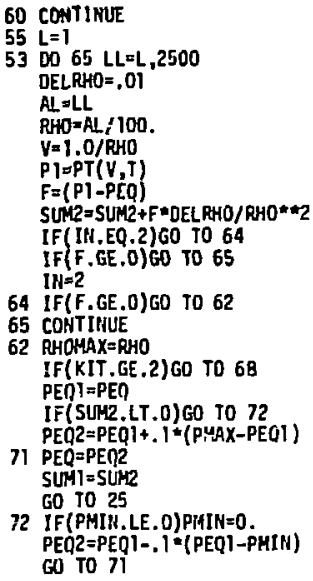

72 IF(PMIN.LE. O) PIAIN=0 . $P E Q 2=P E Q 1-.1$ (PEQI-PHIN) GO TO 71

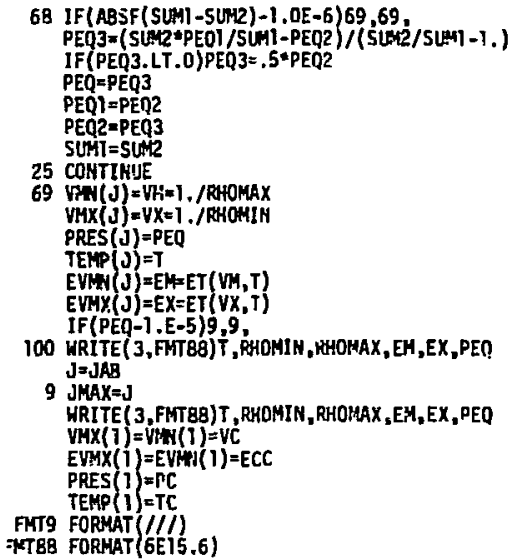

\section{PRESSURE SUBPROGRAM}

\section{ENERGY SUBPROGRAM}

FuACTIOH PT(V.T)

$x=9.38$

TSO=T-T

IF (Y.GT.VJ) 00 TO 2

$x=$ (ves-vi)/vos

$x=0 . x+x$

IF (X.GT.0) 60 TO 12

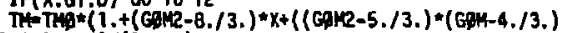

$1-\mathrm{AM})=\mathrm{XSO}) /(1.0-\mathrm{X})=-2$

$12 \prod^{60}$ T0 13

$1-A H) * X 5 Q)$

$135 X=5 * X$

$E G=(C * X) * 2 *(1,+S X / 3,+(S X * 5 X) *(1,-G D H / S) / 6$.

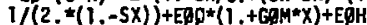

$A X=A M \div X$

GH-GPM-AX

$\mathrm{D}=-2 \cdot * \mathrm{CH}+2 \cdot 13$

$T G=X_{Y N} \star T M$

VOSHX $=$ VPS $\star(1,-X)$

IF(T.GT.TG) 60 TD 15

$E=E Q+T M *(0 S P-.5 * A P)+(G P 2-.5 * A P / T M) \star T S O+3 . * R P \star T$

$P C=(.5 *(G A M A E-G B M+A X) * G P * T S Q-T M *(O S P-.5 * A P *$

$\left.\left.1\left(1 .+T S O / T M^{*}-2\right)\right) *(D+G P H-A X)\right) /$ VOSHX

607016

$15 E=E Q+T H *(D S P-.5 * A P \star(1 .-X M-2))+3.0 * R P \star T$

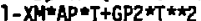

$P C=(.5 *($ GANAAE-GDH+AX)*GP*TSQ-TH*(DSP-.5*AP*

$\left.\left.1\left(1.0-X H^{*}-2+2.0=X H^{\star} T / T H\right)\right) *(D+G P M-A X)\right) / V 95 M X$

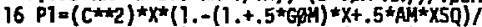

1 (VgSHX $*(1 .-S X) * 2)+($ GPH-AX $) *(E-E Q H) /$ VQSHX $P T=P T+P C+P C C$

RETURA

$2 Z=V B / V$

$2 S Q=2 * 2$

$F P=\left(Z *(T H-Z J) /\left(Z J^{*}(T H-Z)\right)\right) * 3$

ZFAC $=Z *(1, Z Z+Z 50-7 * Z 50) /(1 .-Z) * 3$

$P T=(R P \star T \star Z F A C / V B)-A Y P \approx Z S O /(V B \pm V B)+F P \star(C l+C 2 \star T$

1+C3+TSQ)

RETURY

END
FUACTION ET( $V$, :)

Diso. 38

$T 5 Q=T * T$

IF(V.GT.VI) 60 TO 2

$X=(V D S-V) / V Q S$

$\mathrm{xSQ}=\mathrm{x}+\mathrm{x}$

IF $(X, G T, 0)$ GO TO 12

TH:THP*(1.+(GDUR-8./3.)*X+

$1(($ GQR $-5 . / 3)+.(G 9 M-4 . / 3)-.A M)+X S Q) /(1 .-x)+2$

GD TO 13

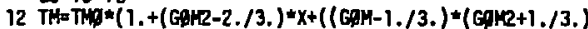

$1-A M)+X S Q 1$

$135 X=5 * X$

$E Q=\left(C^{\star} X\right) \star \star 2 *(1 .+5 X / 3 .+5 X \star 5 X \star(1,-694 / 5) / 6$.

$1 /(2 . *(1 .-5 X))+E g g *(1 .+G Q * * x)+E Q H$

$T G=X M+T M$

IF(T.GT.TG) GO TO 15

$E T=E Q+T H^{\star}\left(D S P-.5^{\star} A P\right)+(G P Z-.5 * A P / T H) * T S Q+3 . * R P \star T$ RETURK

$15 E T=E Q+T M \star\left(O S P-.5 \star A P \star\left(1.0-X M^{\star *} 2\right)\right)+3.0 \star R P \star T$

$1-X H A * A P \star T+G P Z * T S O$

RETURN

$2 Z=\sqrt{B} / \mathrm{V}$

$\mathrm{ZJC}=\mathbf{Z} \mathrm{J}^{*+3}$

$F E=(.5 * V B) *((T H-Z, J) \star 3 *(2 . * 2-2 .+T H) /$

$\left.T\left(Z J C^{*}(T H-Z) * * 2\right)-(T H-Z J) *(2 . * Z J-2 .+T H) / Z J C\right)$ $\mathrm{ET}=.5 * \mathrm{RPD2} * \mathrm{~T}-\left(\mathrm{C} 3^{*} \mathrm{FE}-\mathrm{GP2}\right) \star T S Q-\mathrm{AYP} * \mathrm{Z} / \mathrm{YB}+\mathrm{CI} * \mathrm{FE}+\mathrm{DI}$ RETURN

END 


\section{APPENDIX B}

The following listing is to be inserted into the GRAY EOS subroutine so that the two phase liquid-vapor equilibrium nay be computed. This coding makes use of the critical point and tie line calculations of Appendix $A$ to determine whether an energy-volume point lies inside the two-phase region, and if so, to compute P(V,E). In the GRAY aubroutine used in the WGNDY hydrodynemic code, this insert comes just before the computation of the pressure according to the various parts of GRAY. A complete listing of GRAY as used in WONDY may be found in the report "Themo.
IF(Y.LE. 1,04:VAS) EO TO 124

$E V=E V h X(2)$

If(E,EE.1.2*EV) CO TO 125

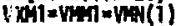

EXMI=EYH)=EVH(i)

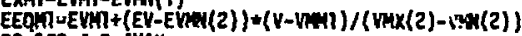

DO $130 \mathrm{Ja2}$, JMAX

$v x-\operatorname{vin} x(\mathrm{~d})$

EX-EVMX (j)

Eit=Vin(d)

EVaEVW (J)

$E E Q * E V+(E X-E V) *(V-V H) /\left(V X-W^{2}\right)$

IF E.LT.EEQ) 60 To I3i

$E B H=E V+(E V H-E V) *(V-V H) /(V I N-V H)$

IF(E.GE,EBH) $G 0$ TO 125

$E B L-E X+(E X-E X M) *(Y-\psi X) /(4 X-i X M T)$

IF(E-EBL) $133,125,125$

131 EEQAT-EEO

$v x=1=4 x$

$V$ VIT $=V M$

EXYT=EX

EVT) $=$ EV
Mechanical Response of Porous Carbon" (A.C. Holt, A.S. Kusubov, D.A. Young and W.H. Gust, LLL Rpt. UCRL-51330 (1973)). The insert goes between the statemerits (p. 67 of the above report) "IF(CARBON. EQ.1) GO TO 100" and "IF(E.LE.EM2) GO TO MELT". The two statements lying between these statements in the report are included in the insert in modified form. Once again, if several materials an being considered, the tic line parameters must be subscripted according to the material layer.

130 conrthute

J=JMAX

EQL $=E X+W X / V$

IF(E. GE.EBL) CO TO 125

SLEH $x-E V /(0.96 / V D S-1.0 / V H)$

EBH=EY+SLEN+ $(1,0 / Y-1,0 / V M)$

IF(E. GE.EBH) GO TO 124

$T E M=T E P(J-1)$

TEXTEMP(J)

$T=(E / E E Q H)) *(T E-300)+$.300 .

$5 T=$ BHYAPOR

GO TO 134

133 ST=8HYAPOR

ERAT-(EEQHT-E)/(E-EEQ)

TE $=\operatorname{TEMP}(j)$

TEMI $-T E H P(J-1)$

$T=(T E \cap E R A T+T E H) /(1,+E R A T)$

$134 \mathrm{PQ}=\mathrm{PRES}\{\mathrm{J}\}$

SLPR $=\operatorname{LOGF}(\operatorname{PRES}(\mathrm{J}-1) / P Q) /(1 . / \mathrm{TEMT}-1 . / \mathrm{TE})$

$P S=P Q+E X P F(S L P R+(1.0 / T=1.0 / T E))$

60 TO RTH

125 IF(X.LT.XJ) GO TO VAPR

124 IF(E.LE.EMI) GO TO SOLID 


\section{APPENDIX C}

A loz log plot of pressure-density isotherms is shown here for exch of the sixteen metals in the GRAY report (Ref. 1). The liquid-vapor region is empharized. The temperatures of the adjacent isothems are $2000^{\circ} \mathrm{K}$ spart and the highest and lowest isothems are labelled in degrees Kelvin. Zigzags in the high density isothems represent melting. Mismatching of isotherms at the twophose boundery may be seen near the critlcal point in some of the ploth. 


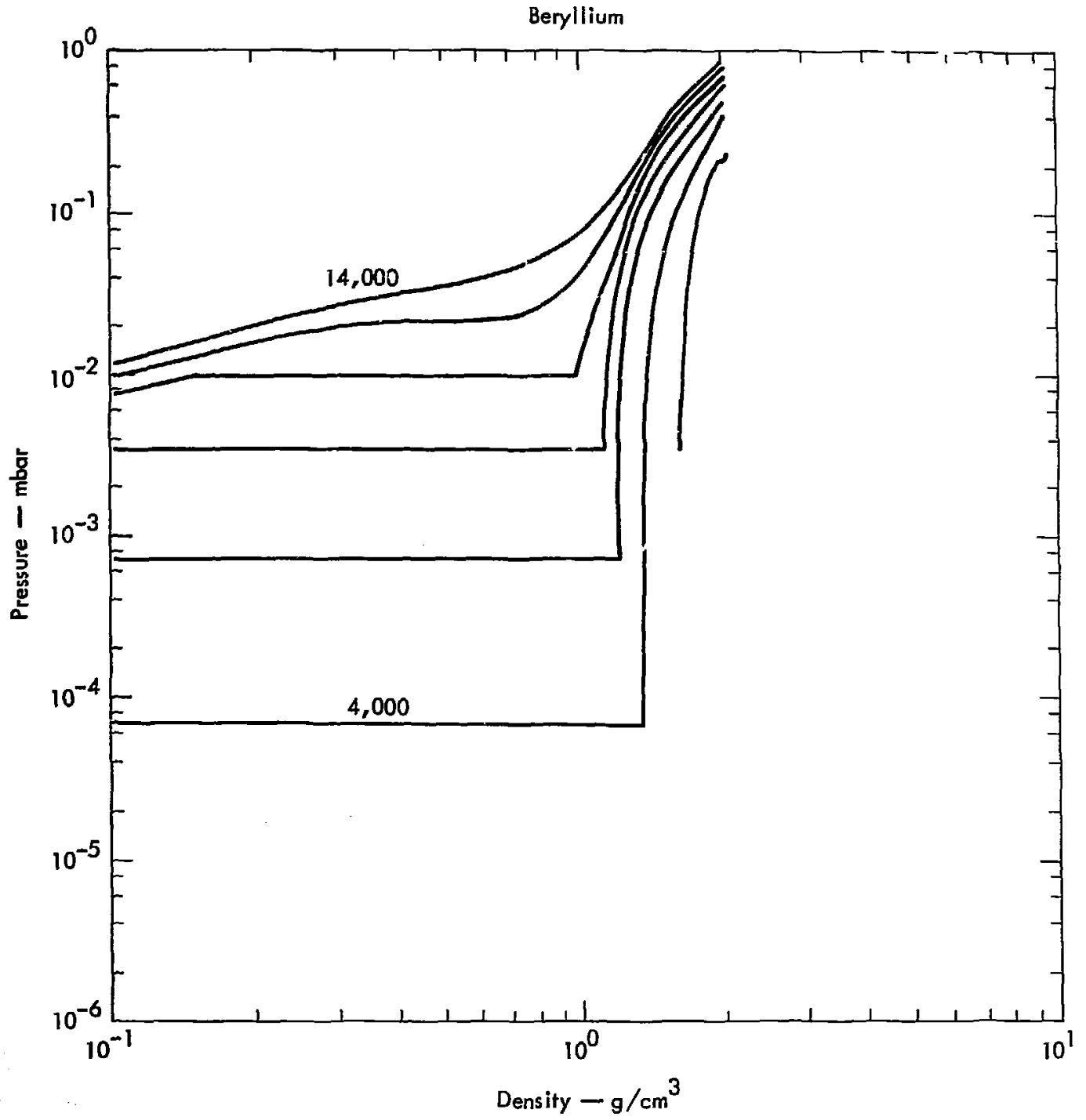




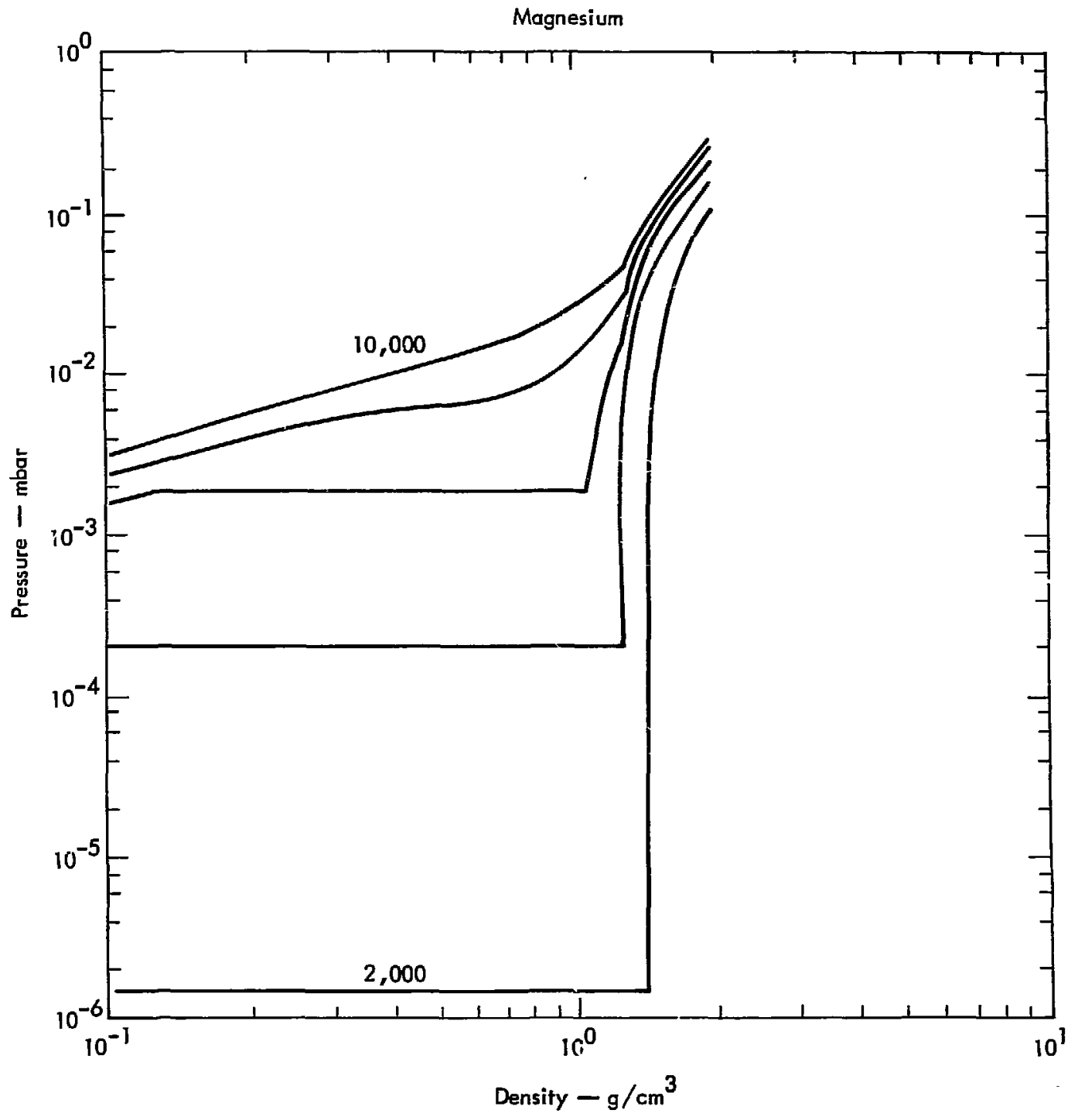




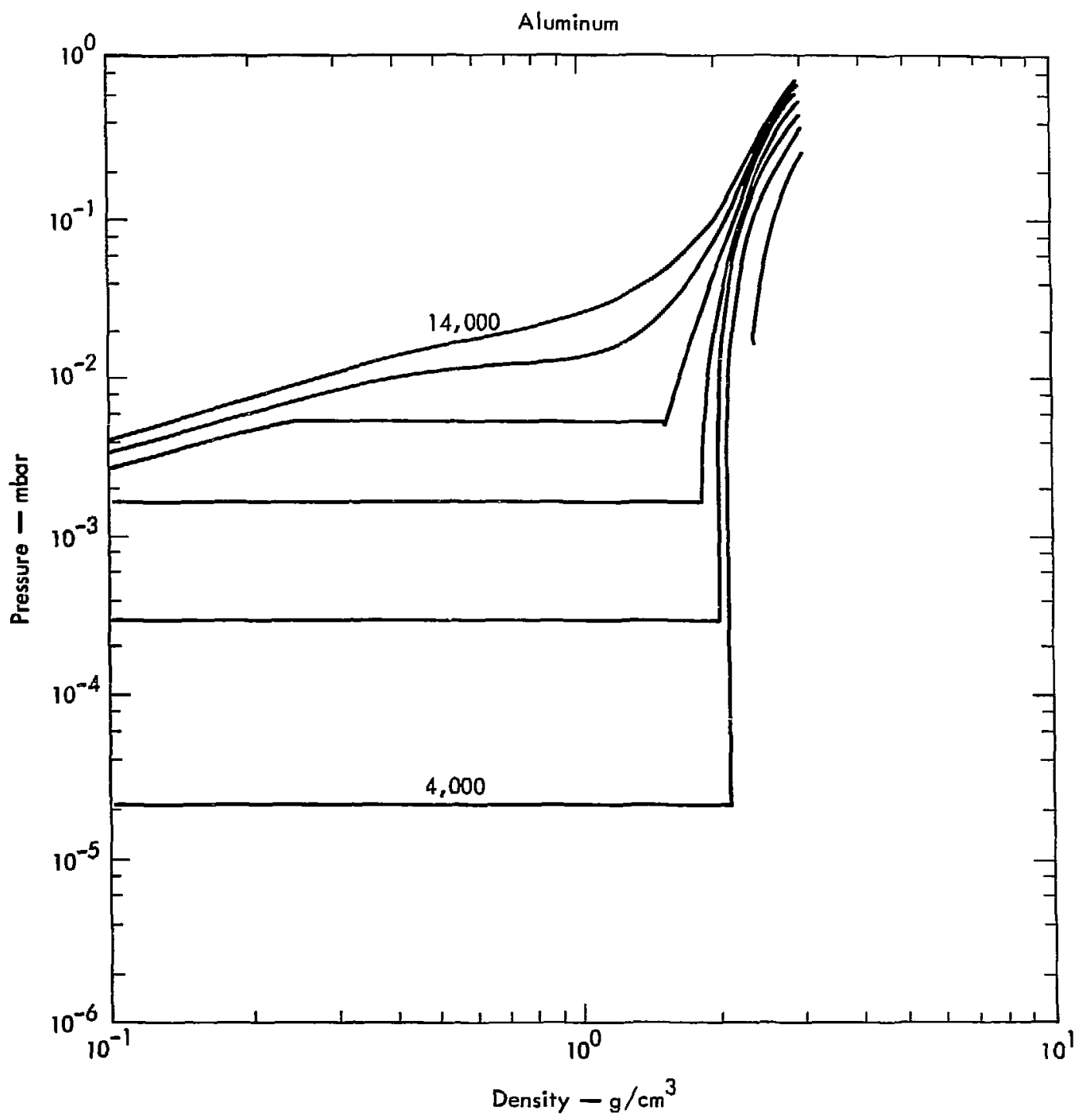

.18. 
Titanium

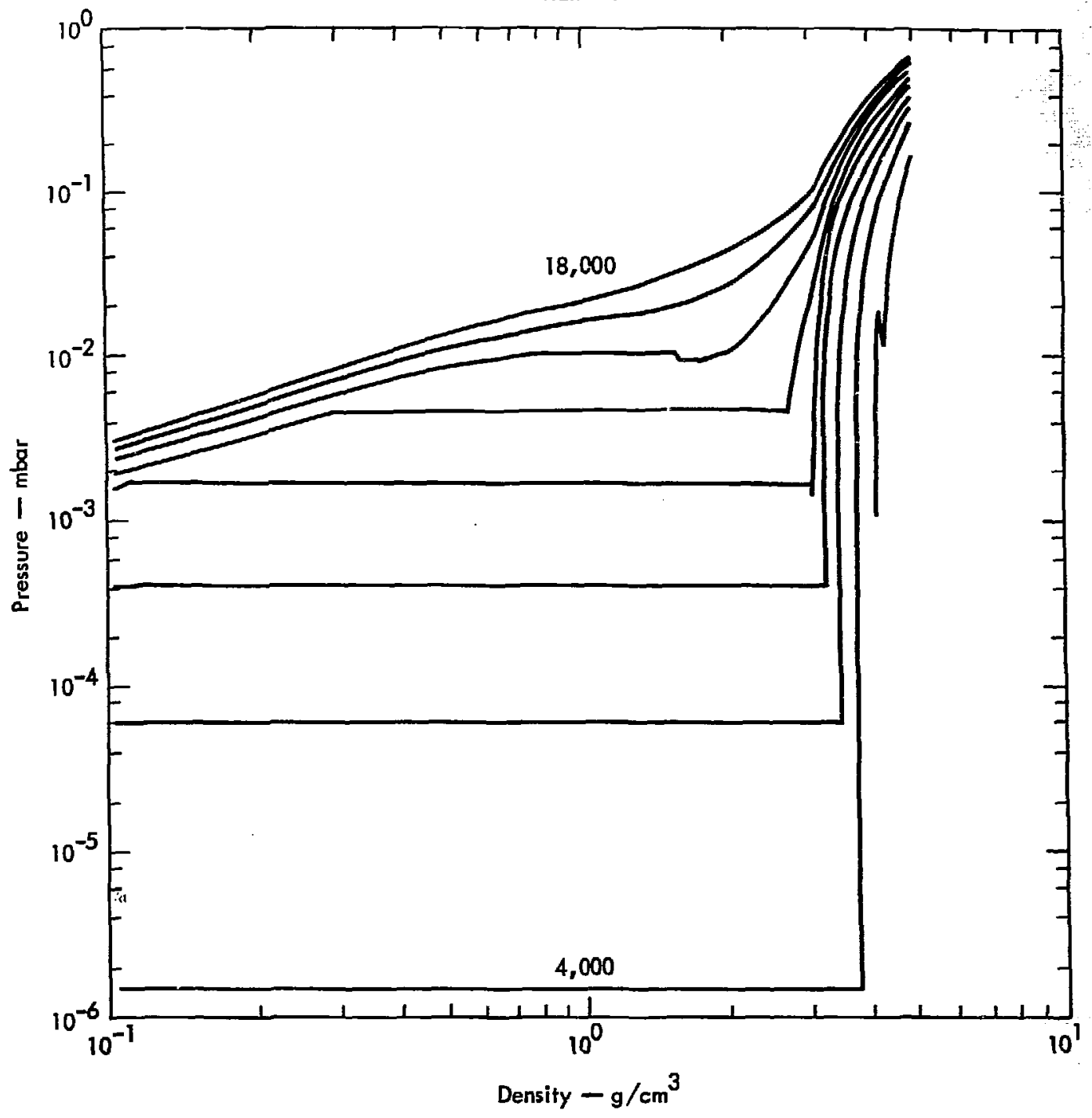




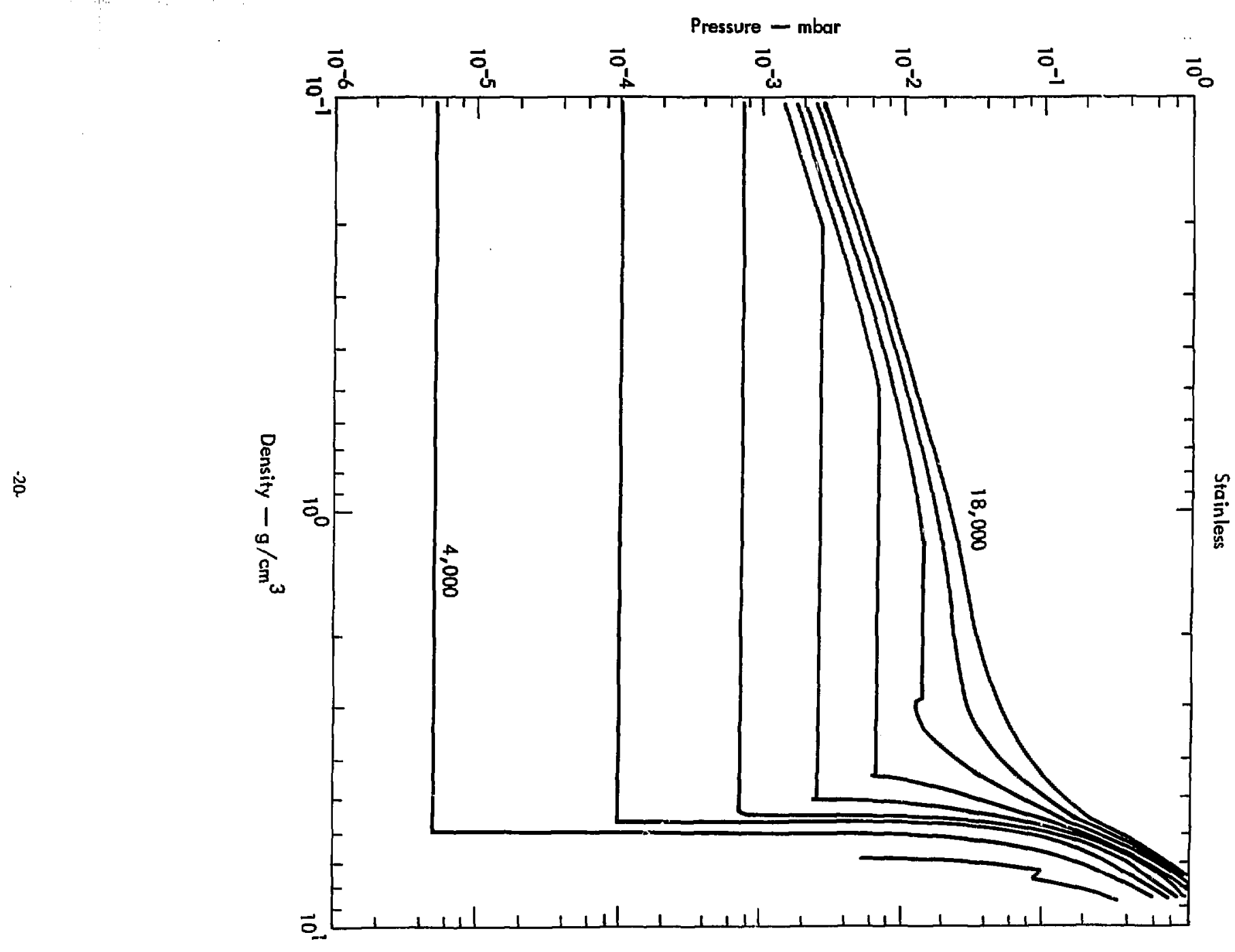




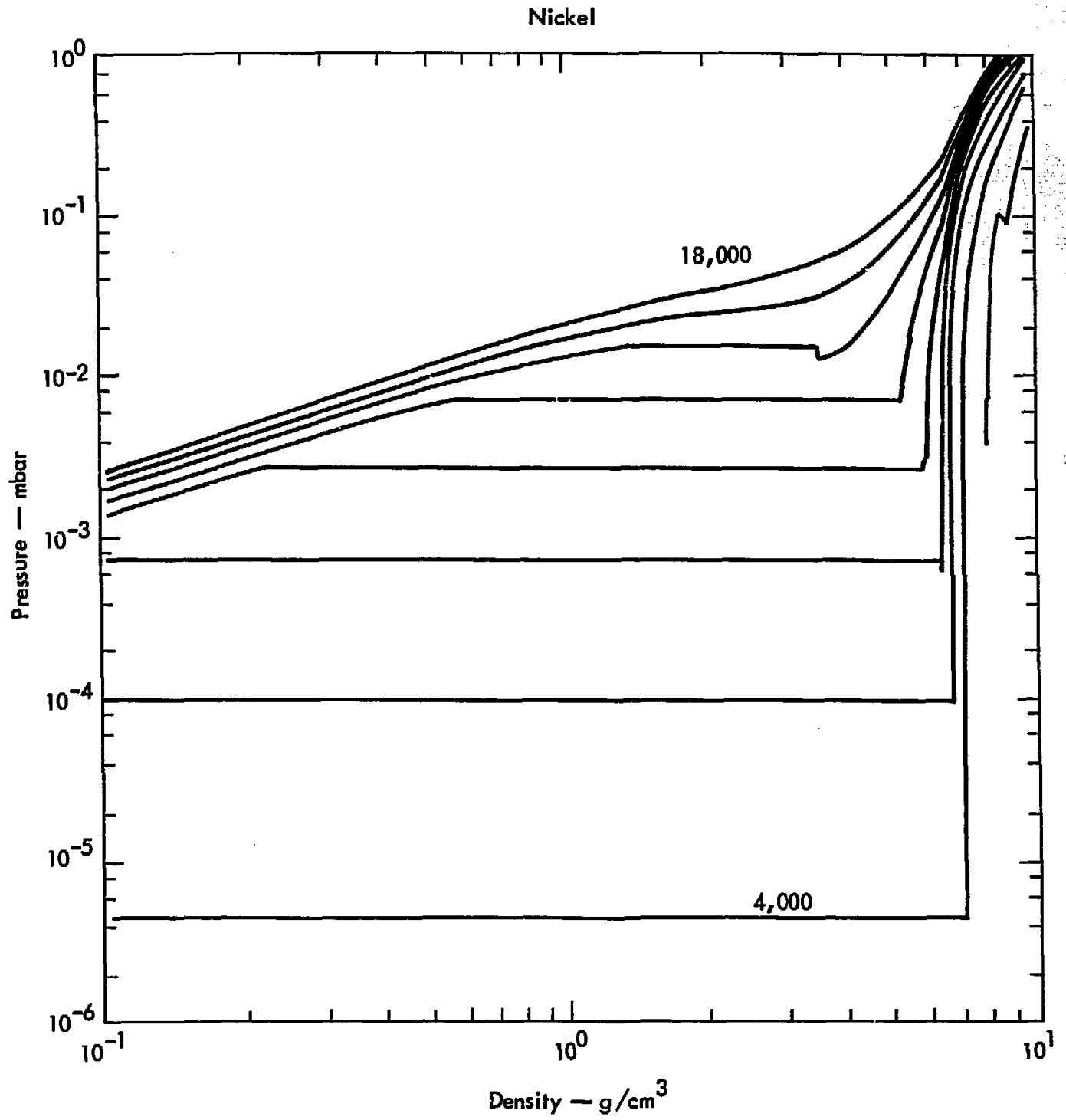


Monel

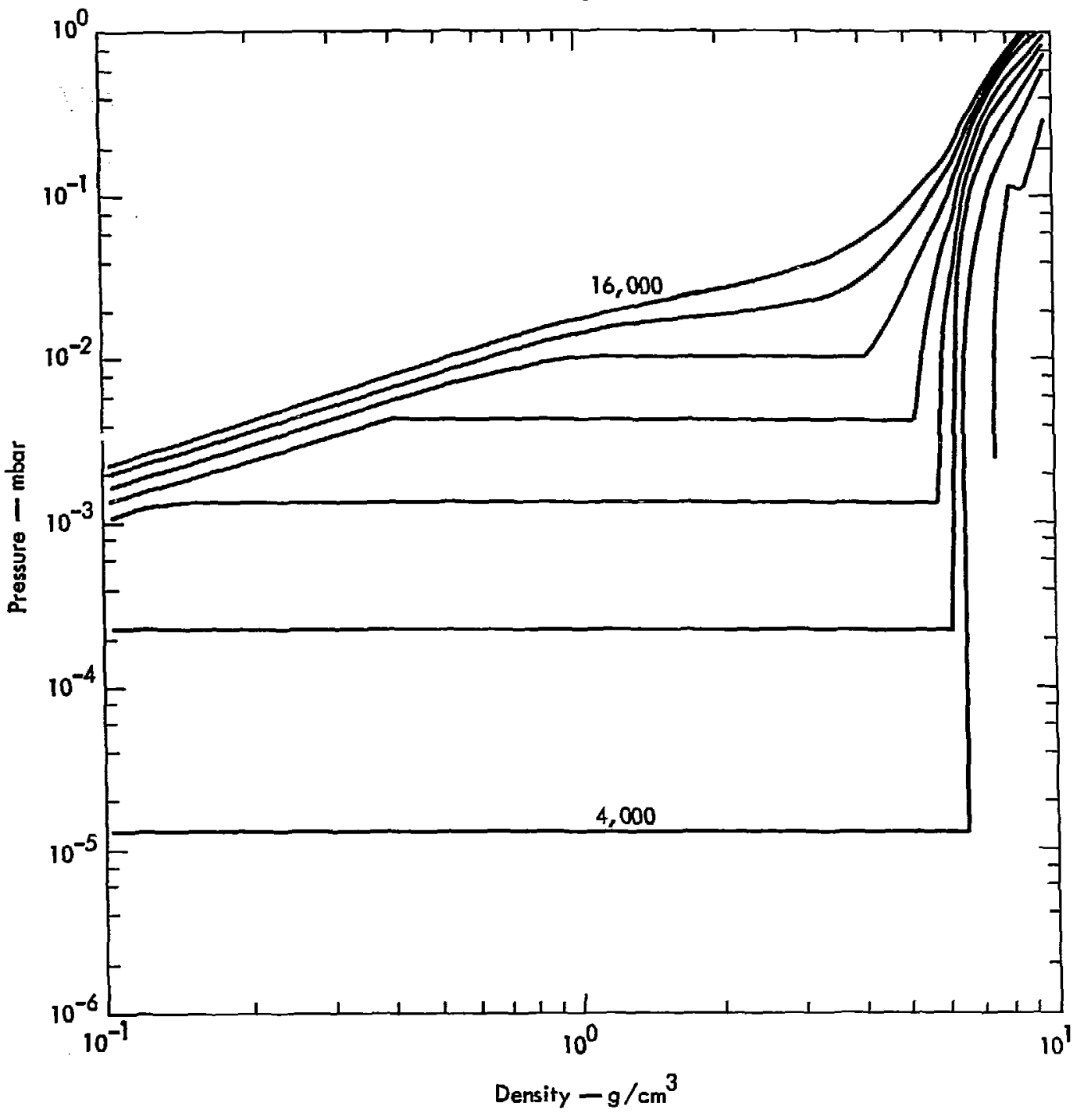




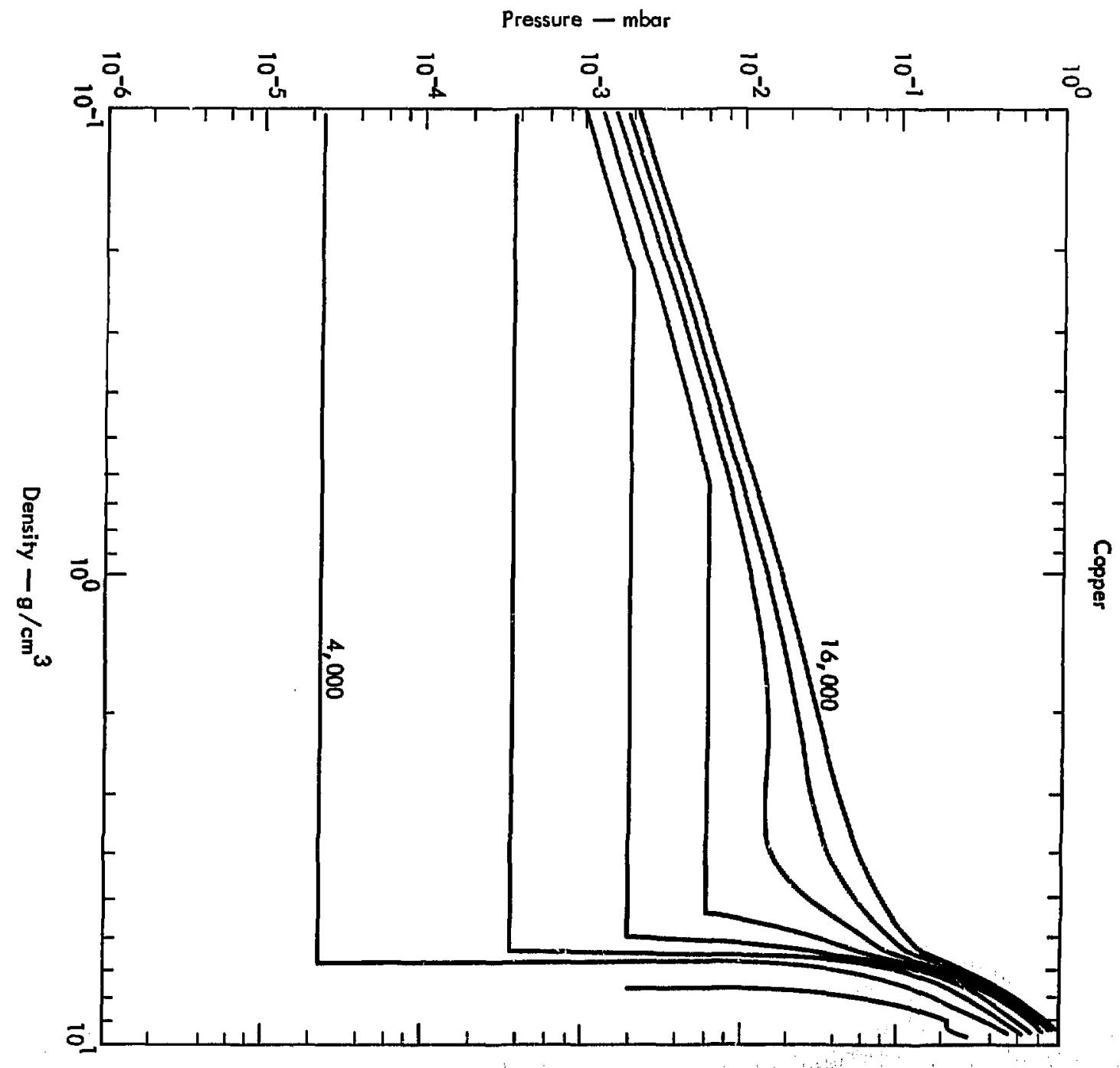




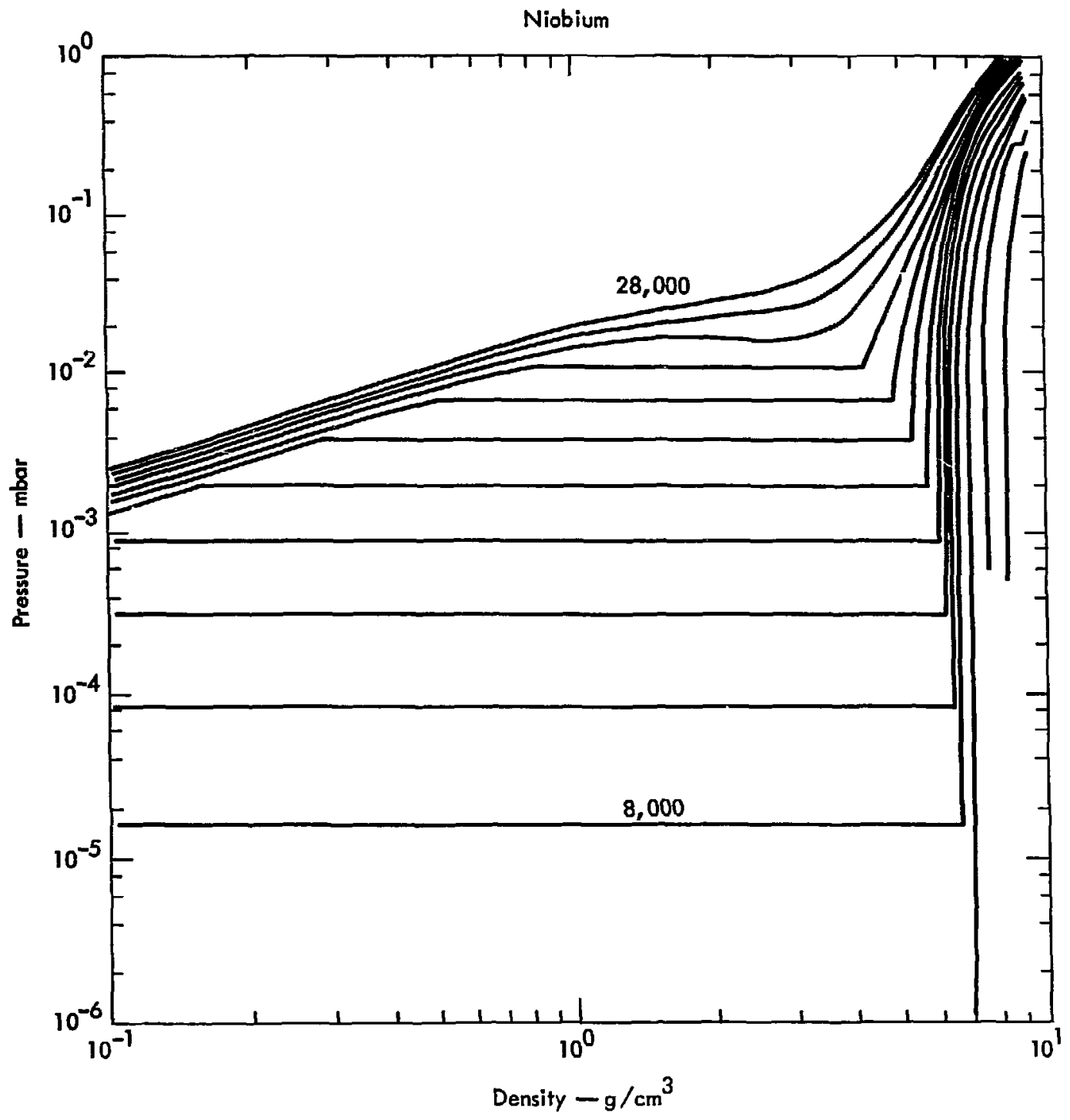




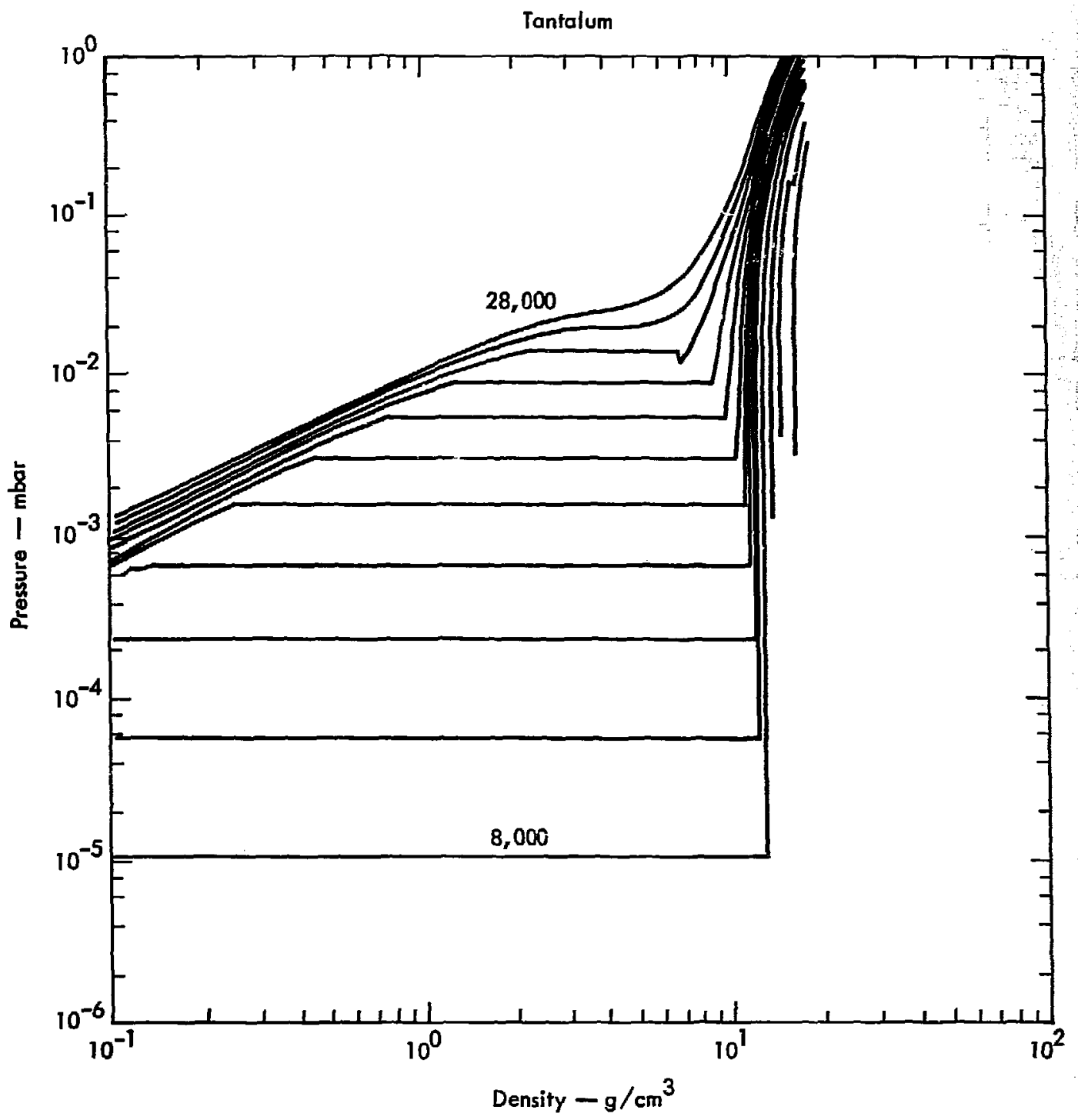


Tungsten

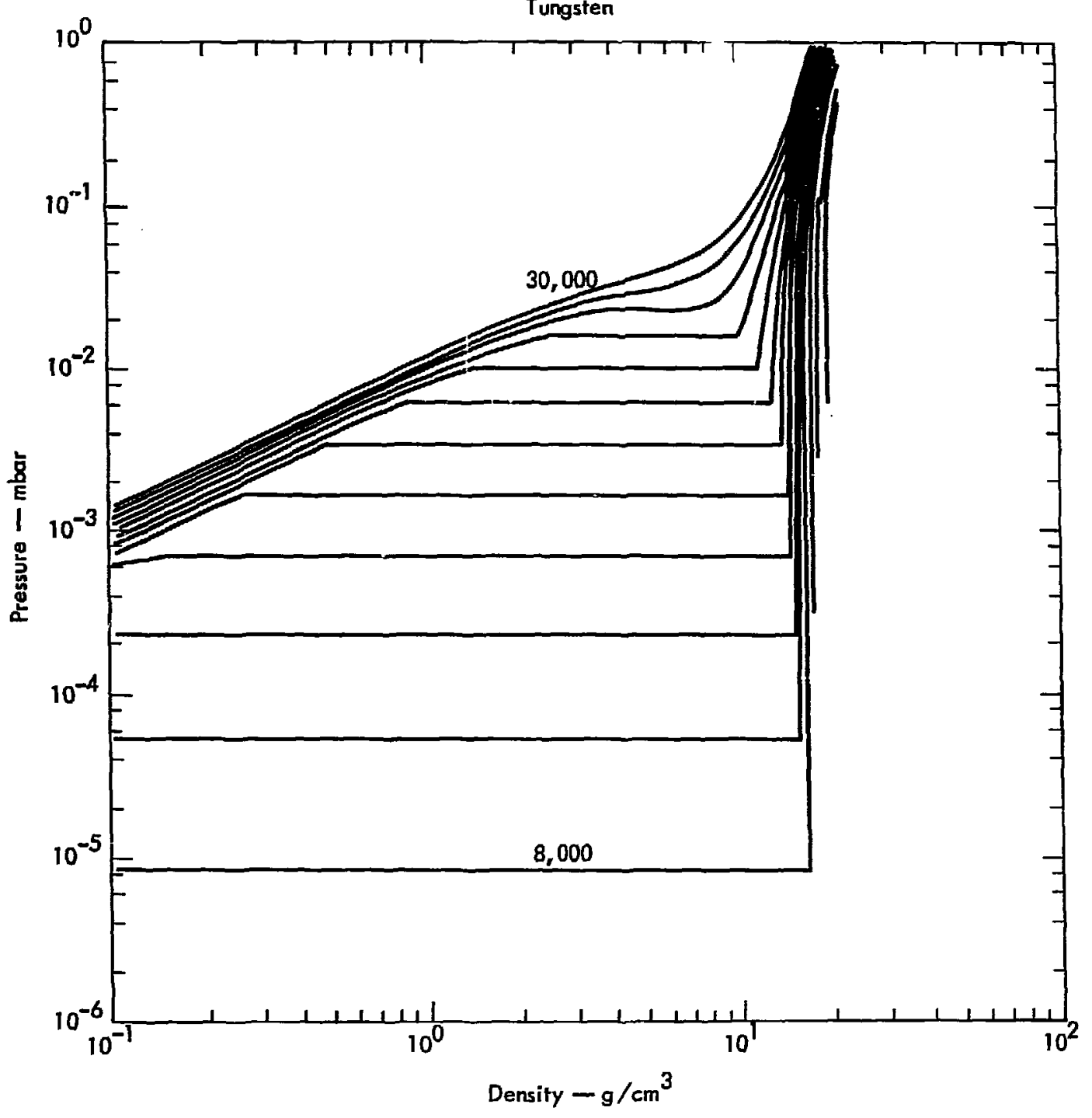




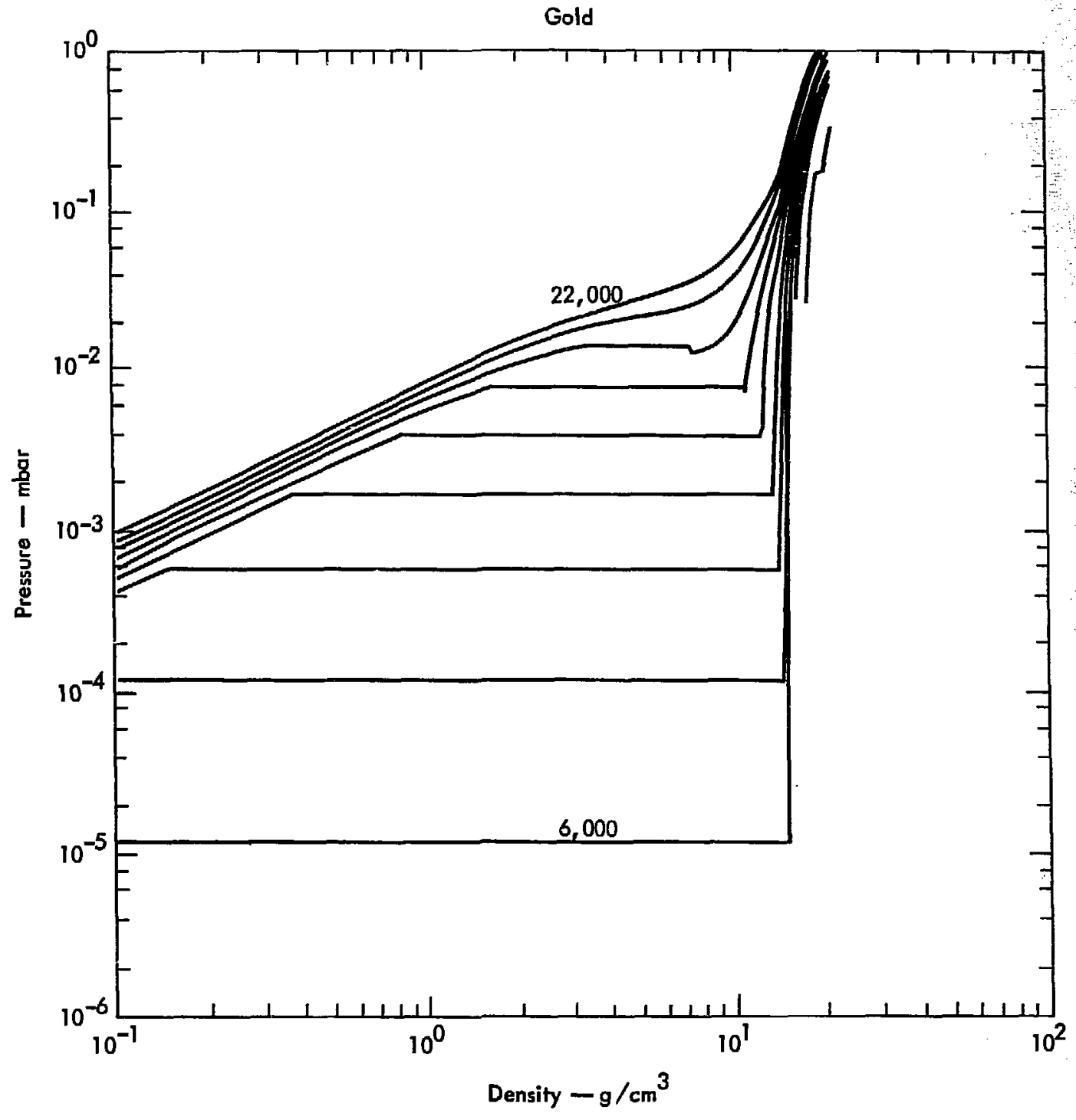




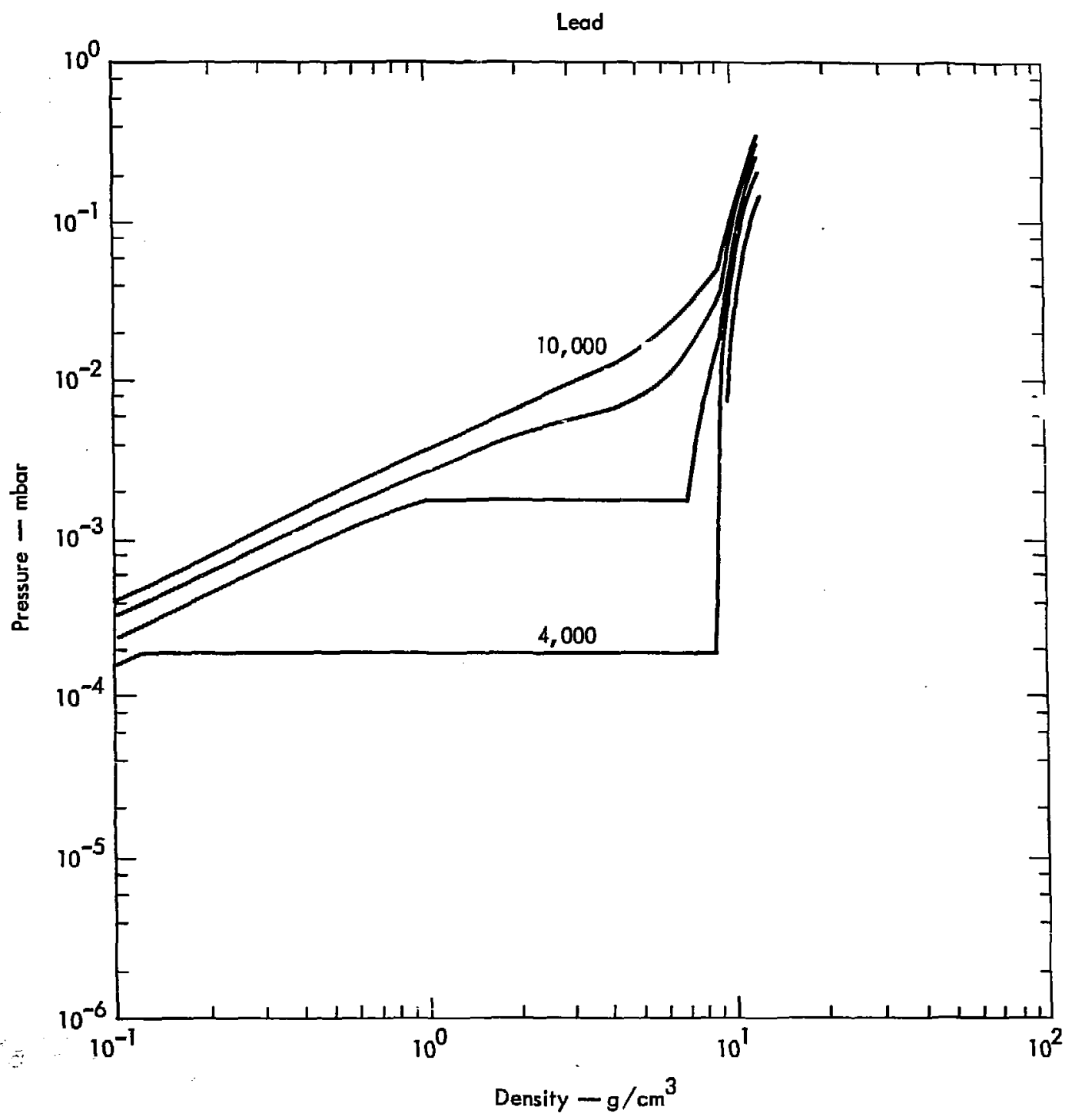

$-28-$ 


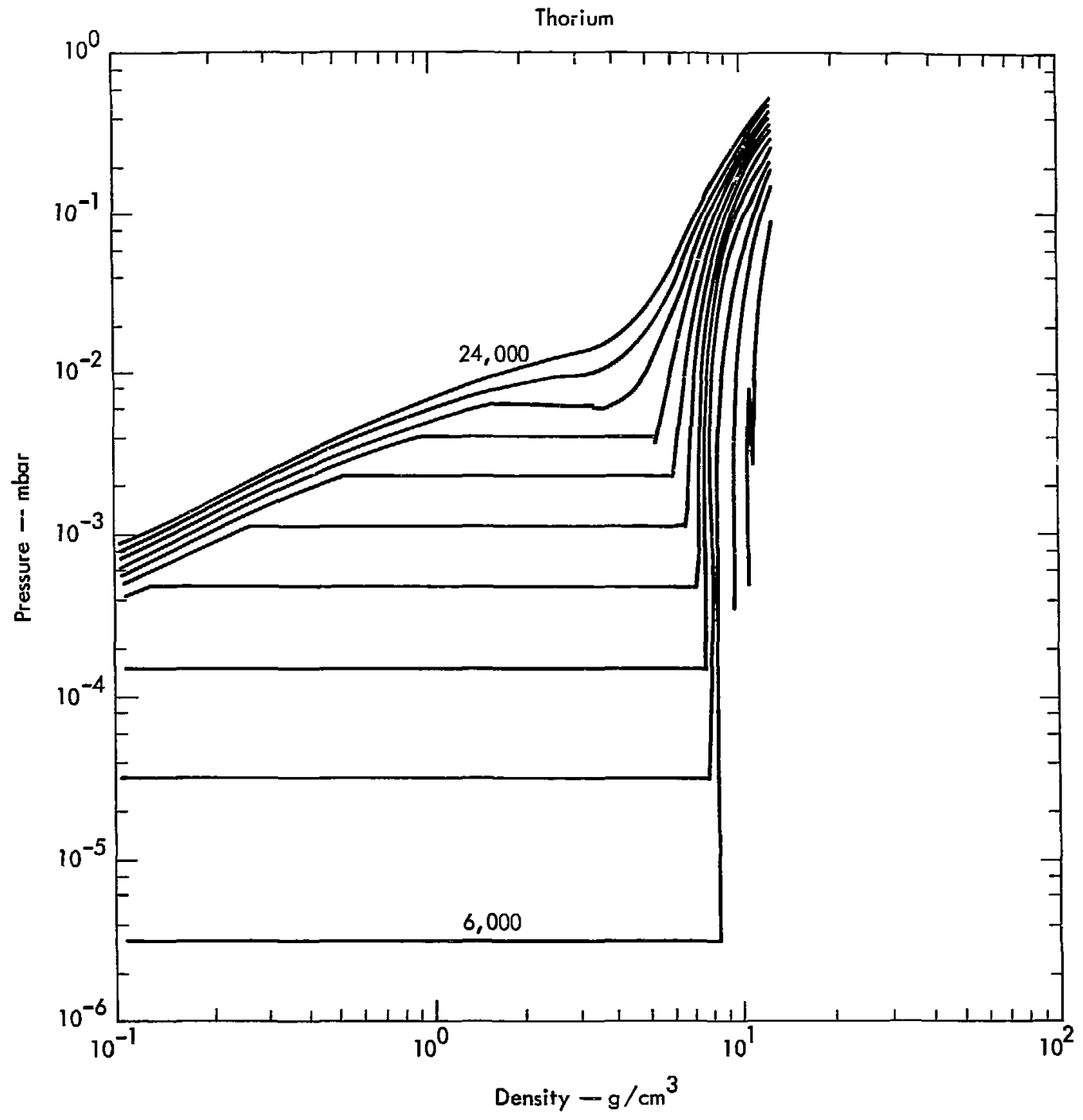




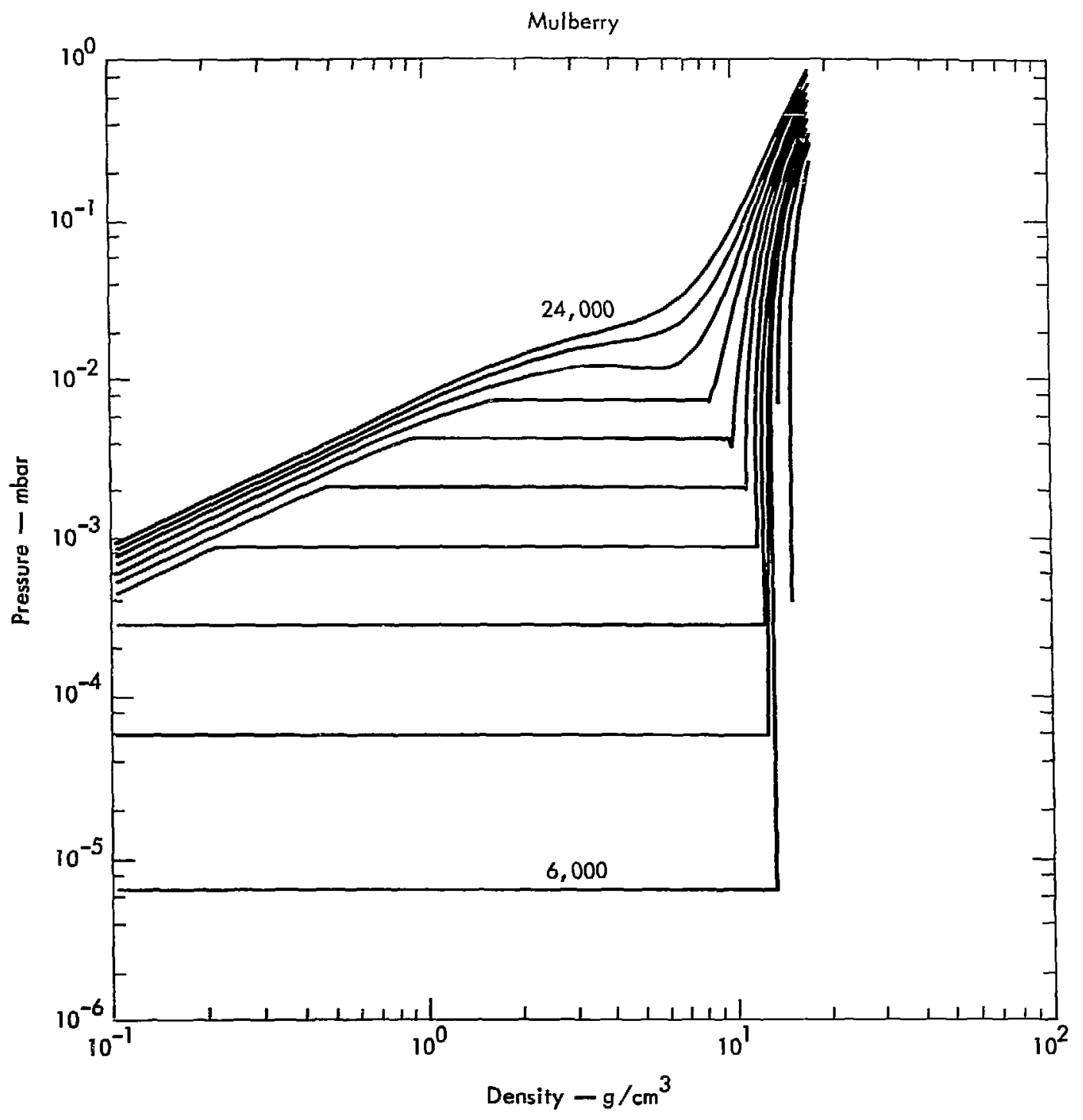




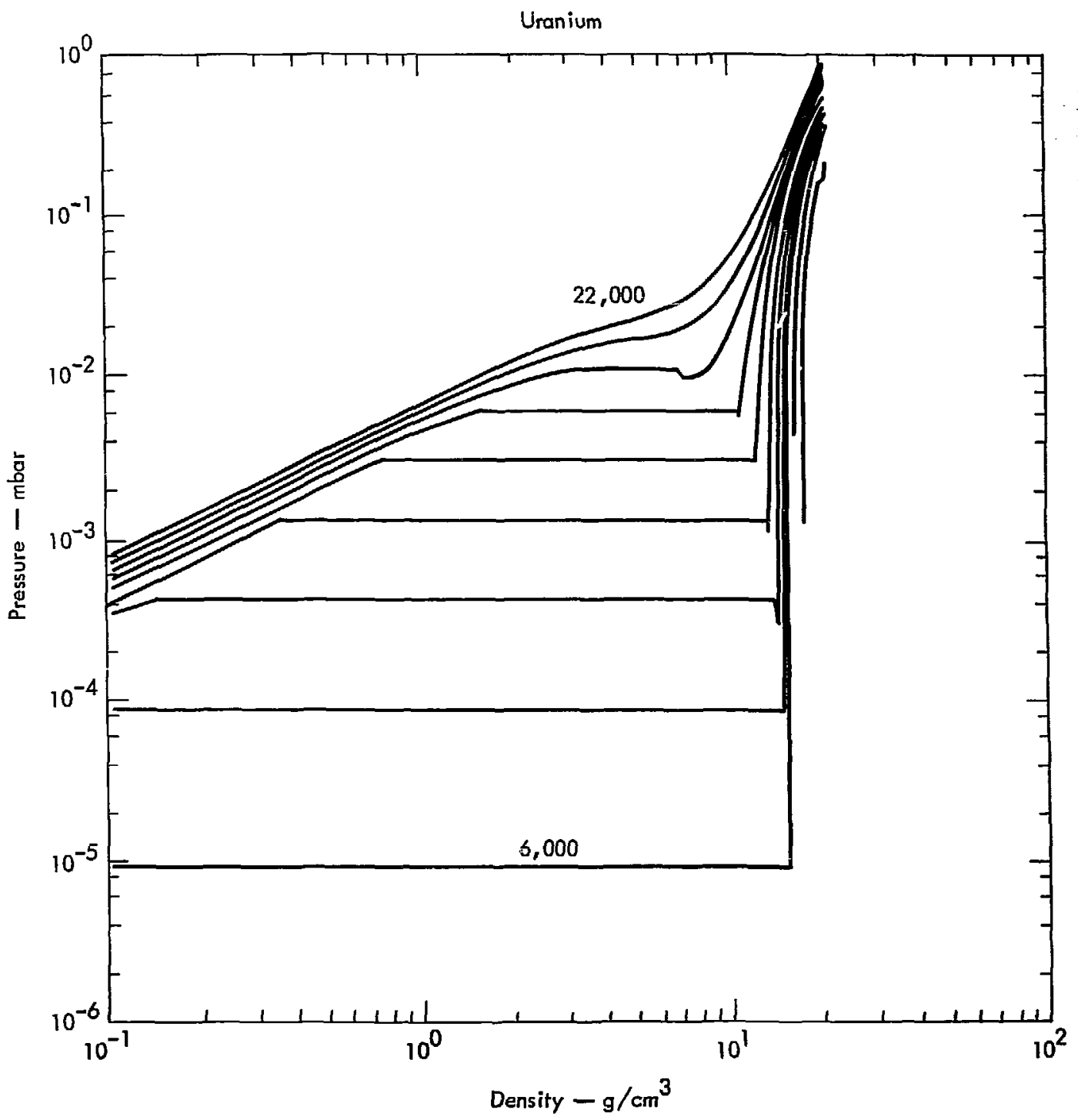

